\title{
Explicit parametric solutions of lattice structures with Proper Generalized Decomposition (PGD)
}

\author{
Applications to the design of 3D-printed architectured \\ materials
}

\author{
Alberto Sibileau • Alberto \\ García-González • Ferdinando \\ Auricchio · Simone Morganti • Pedro \\ Díez
}

Received: date / Accepted: date

\begin{abstract}
Architectured materials (or metamaterials) are constituted by a unit-cell with a complex structural design repeated periodically forming a bulk material with emergent mechanical properties. One may obtain specific macroscale (or bulk) properties in the resulting architectured material by properly designing the unit-cell. Typically, this is stated as an optimal design problem in which the parameters describing the shape and mechanical properties of the unit-cell are selected in order to produce the desired bulk characteristics. This is especially pertinent due to the ease manufacturing of these complex structures with 3D printers. The Proper Generalized Decomposition (PGD) provides explicit parametic solutions of parametric PDEs. Here, the same ideas are used to obtain parametric solutions of the algebraic equations arising from lattice structural models. Once the explicit parametric solution is available, the optimal design problem is a simple post-process. The same strategy is applied in the numerical illustrations, first to a unit-cell (and then homogenized with periodicity conditions), and in a second phase to the complete structure of a lattice material specimen.
\end{abstract}

Keywords Parametric lattice structure · Proper Generalized Decomposition • Explicit parametric solution $\cdot$ architectured materials $\cdot 3 \mathrm{D}$ printing

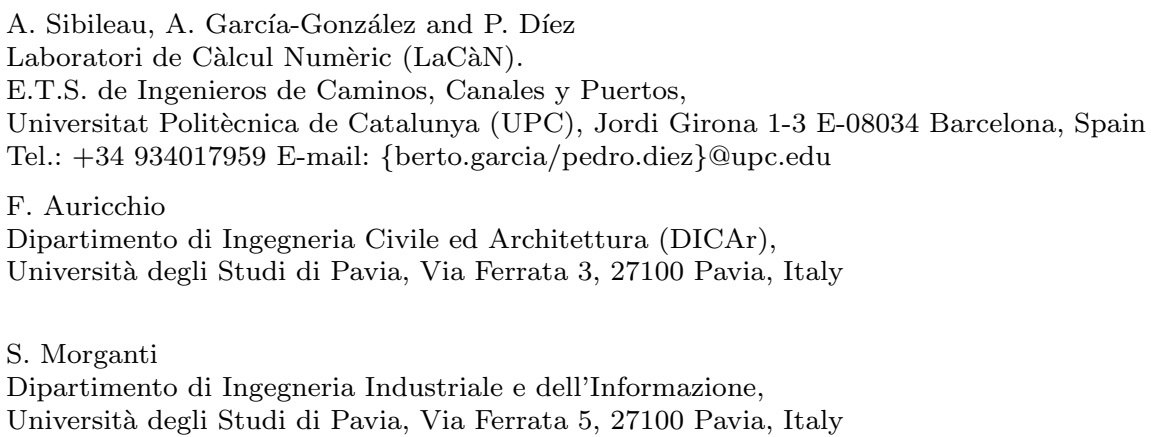




\section{Introduction}

Architectured materials (or metamaterials) are built as tessellations of smallscale structures (also named as cells). The cells are designed to obtain specific physical properties of the bulk. This concerns often the mechanical response, which is the scope of this work, but not only. The same concept is also used to obtain astonishing thermic, acoustic, optic or electromagnetic properties, see for instance [5]. Thus, as stated in [26], scientific and technical communities trend toward obtaining novel macro-structural material responses via micro-structural design. Achieving these new material properties is expected to highly impact the design of new devices in many fields of science and technology, see $[8,10]$. Just to mention a few applications, the use of architectured materials results in negative or null thermal expansion coefficients [30,15], pentamodes or simile-fluid solids $[21,17,2]$, elastic buckling or snapping based metamaterials $[25,23]$, negative index of optical refraction $[16,13]$ and materials with negative Poisson's ratio $[7,14,24]$, also known as auxetic materials.

A natural problem arising in this context is the design of an architectured material with tailored properties. This results in an inverse problem consisting in identifying the design parameters of the cell producing the desired properties in the bulk material. This is already addressed by the early works of Sigmund $[28,29]$ in the context of computational mechanics with homogenization. The main difficulty of handling a parametric description of the micro-structure and to determine its influence in the emerging properties of the bulk is the multidimensional character of the problem. The computational complexity in-

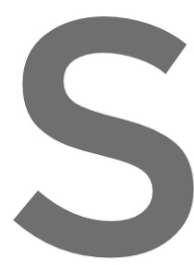
creases exponentially of the inverse problem. and making progresses strengthen our physica

A particular and intere metamaterials with additir
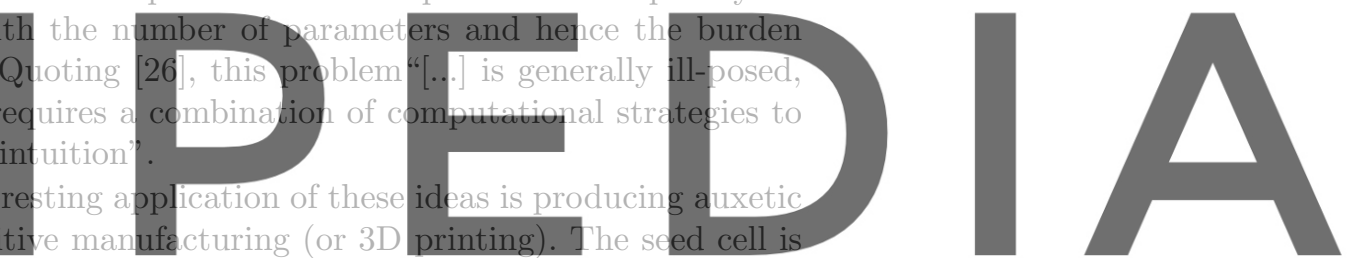

in this case a 3D printed lattice material with parametrically described shape

Register fand size. In this context. different numerical models testing divers scenarios a

bulk. For instance, taking an inverse honeycomb configuration, first proposed

by [1] and extensively analyzed later by $[27,12,18]$. However, as pointed out above, the computational burden is still a bottleneck when the number of parameters is moderately large.

One possible solution to affordably deal with the so-called curse of $d i$ mensionality is using Reduced Order Models (ROM). Here, we focus on a particular ROM: the Proper Generalized Decompostion (PGD), presenting a separable approximation computed using a combination of a greedy algorithm (to compute successively the terms) and an alternated directions scheme (to compute iteratively the modes in each term), see $[9,32]$. This technique is specially well suited to simulate the behavior of the parametric structure (with parameters describing both geometrical and material properties of the cell) because it provides an explicit parametric solution, also denoted as computa- 
tional vademecum. In the PGD philosophy, the computational vademecum is obtained offline, often using High Performance Computing resources. Then, to solve the inverse problem, the parametric design space is browsed as a post-process, in an online phase providing real-time responses.

This paper aims at presenting a new PGD formulation to efficiently deal with the parametric lattice material problem. The explicit parametric solution (computational vademecum) is to be exploited to derive the macro-scale properties of the resulting architectured material an hence to efficiently solve the inverse problem under consideration.

The remainder of the paper is structured as follows. Section 2 describes the parametric discrete problem and the functional setup. Section 3 introduces in detail the generalization of the Proper Generalized Decomposition to the case of intrinsically discrete lattice structures. Then, in Section 4 the proposed strategy is applied to solve the parametric model in different structural configurations. Some concluding remarks are drawn in Section 5.

\section{Problem statement: parametric lattice structure}

The shape and material properties of a lattice structure are characterized by $\mathrm{n}_{\mathrm{p}}$ parameters $\mu_{i}$, for $i=1,2, \ldots, \mathrm{n}_{\mathrm{p}}$, each ranging in a real interval $I_{i} \subset \mathbb{R}$. For the sake of shortening the notation, the $\mathrm{n}_{\mathrm{p}}$ parameters are collected in a vector $\mu=\left[\mu_{1} \mu_{2} \ldots \mu_{\mathrm{n}_{\mathrm{p}}}\right]^{\top}$. Accordingly, $\mu$ ranges in the multidimensional

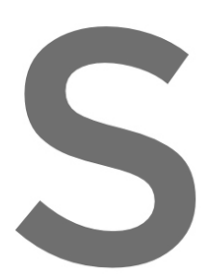
parametric space $\mathcal{D}=1$

The deformation of displacements (nodal cis known of the parametric problem is denoted by $\mathrm{U}(\mu) \in \mathbb{R}^{\mathrm{n}}$
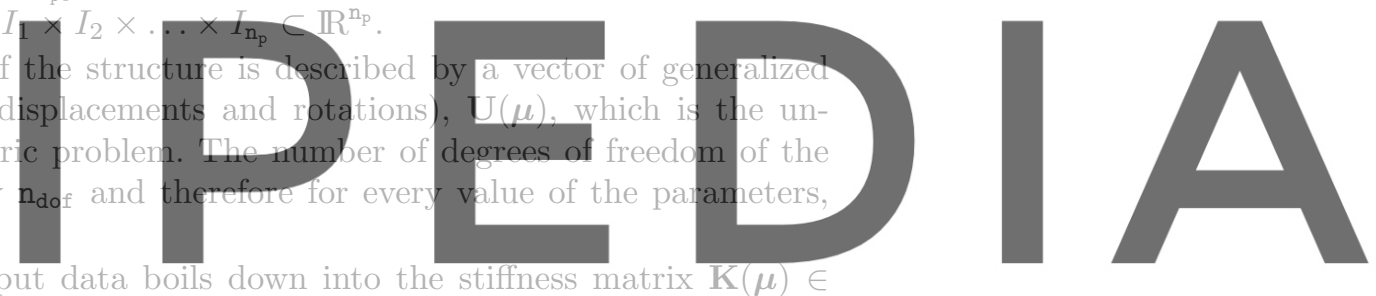

The parametric input data boils down into the stiffness matrix $\mathbb{K}(\mu) \in$

$\mathbb{R}^{n_{\text {dof }} \times n_{\text {dof }}}$ and the vector of generalized nodal forces (including forces and mo-

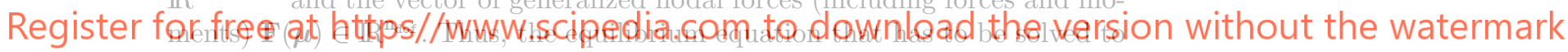
obtain $\mathrm{U}(\mu)$ reads

$$
\mathbf{K}(\boldsymbol{\mu}) \mathbf{U}(\boldsymbol{\mu})=\mathbf{F}(\boldsymbol{\mu})
$$

Equation (1) is stated in a parametric fashion, in the sense that input data $\mathbf{K}$ and $\mathbf{F}$ depend on $\boldsymbol{\mu}$ and therefore also the solution $\mathbf{U}$ depends on $\boldsymbol{\mu}$. Thus, all these mathematical objects are fields taking values in the multidimensional parametric space $\mathcal{D}$. In the following it is assumed that the parametric dependence of the input data is regular enough to be square integrable, that is $\mathbf{F}(\boldsymbol{\mu}) \in\left[\mathcal{L}_{2}(\mathcal{D})\right]^{\mathrm{n}_{\mathrm{dof}}}$ and $\mathbf{K}(\boldsymbol{\mu}) \in\left[\mathcal{L}_{2}(\mathcal{D})\right]^{\mathrm{n}_{\mathrm{dof}} \times \mathrm{n}_{\mathrm{dof}}}$. Note that the parametric functional space $\mathcal{L}_{2}(\mathcal{D})$ is also expressed in terms of the sectional spaces $\mathcal{L}_{2}\left(I_{i}\right)$, $i=1,2, \ldots, \mathrm{n}_{\mathrm{p}}$. Namely, $\mathcal{L}_{2}(\mathcal{D})=\mathcal{L}_{2}\left(I_{1}\right) \otimes \mathcal{L}_{2}\left(I_{2}\right) \otimes \cdots \otimes \mathcal{L}_{2}\left(I_{\mathrm{n}_{\mathrm{p}}}\right)$ which means that freezing all the parameters but one, the remaining functional dependence is square integrable. 
Matrix $\mathbf{K}$ is build up assembling the contribution of all the beam elements of the lattice structure. The formulation used in the numerical examples correspond to Bernoulli beam elements. However, all the developments are valid for any beam or structural element formulation. The parameters included in $\boldsymbol{\mu}$ do characterize the geometry and material properties of the individual elements. The same concept is readily generalized to the parameterization of substructures that are also assembled into the global structure. This strategy is particularly useful in the context of architectured materials, in which a unit-cell is periodically replicated in the bulk structure.

Equation (1) is also expressed in integral form. First, the residual of (1) is introduced as

$$
\mathbf{R}(\mathbf{U}(\boldsymbol{\mu})):=\mathbf{F}(\boldsymbol{\mu})-\mathbf{K}(\boldsymbol{\mu}) \mathbf{U}(\boldsymbol{\mu}) .
$$

Thus, using the weighted residuals idea, one can state that $\mathbf{U}(\boldsymbol{\mu})$ is the solution of (1) if and only if

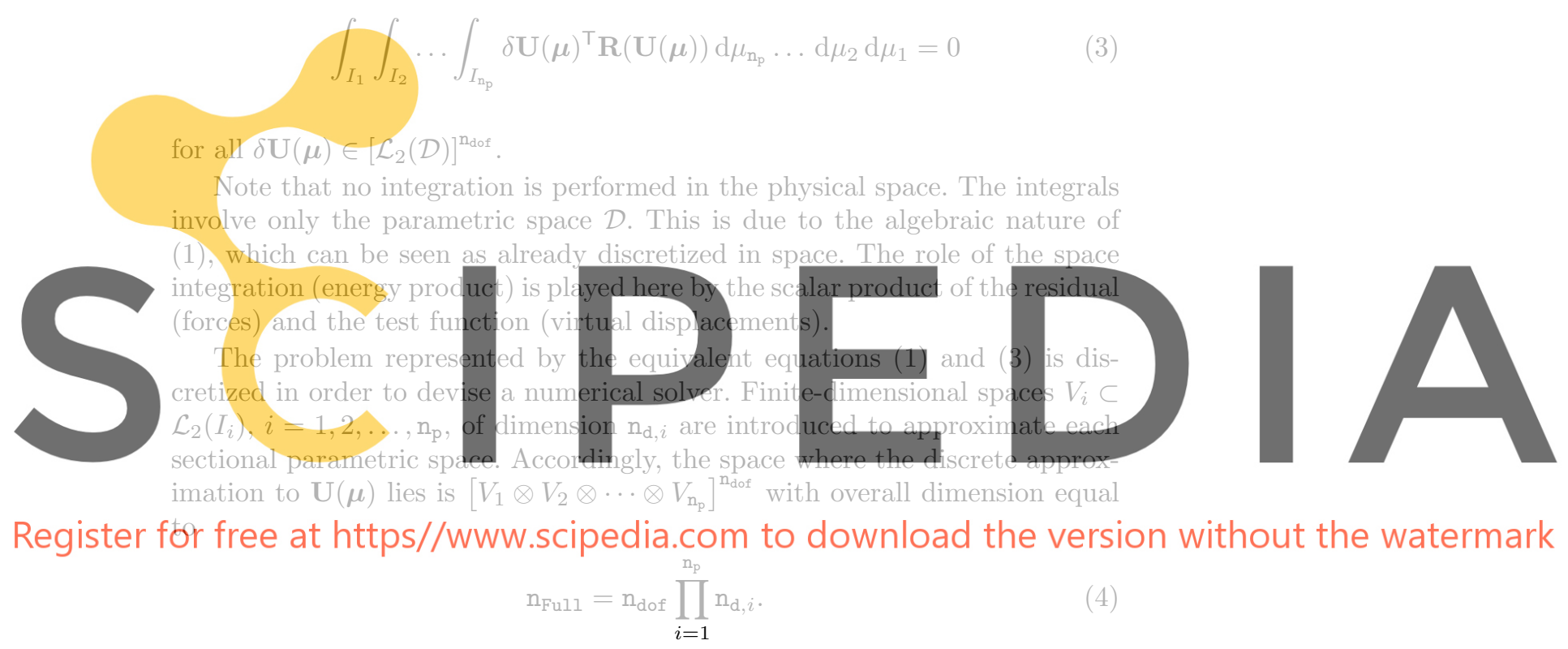

The total number of unknowns $\mathrm{n}_{\mathrm{Full}}$ is growing fast with the number of parameters $\mathrm{n}_{\mathrm{p}}$, producing the so-called curse of dimensionality. In other words, the number of degrees of freedom in the full multidimensional problem, $\mathrm{n}_{\mathrm{Full}}$, grows exponentially with $\mathrm{n}_{\mathrm{p}}$.

Reduced Order Models (ROM) are possible alternatives to overcome this difficulty. In particular, the Proper Generalized Decomposition (PGD) is a ROM based on the idea of providing a separable decomposition of the multidimensional function and therefore reducing the exponential computational complexity into a linear one. Next section discusses the application of PGD to the structural problem under consideration. 


\section{Proper Generalized Decomposition solver}

3.1 Separable approximations

The unknown $\mathbf{U}(\boldsymbol{\mu})$ is to be approximated by a separable approximation $\mathbf{U}_{\mathrm{PGD}}^{n}(\boldsymbol{\mu})$ with $n$ terms, namely

$$
\begin{aligned}
\mathbf{U}(\boldsymbol{\mu}) \approx \mathbf{U}_{\mathrm{PGD}}^{n}(\boldsymbol{\mu}) & =\sum_{m=1}^{n} \mathbf{u}^{m} G_{1}^{m}\left(\mu_{1}\right) G_{2}^{m}\left(\mu_{2}\right) \ldots G_{\mathrm{n}_{\mathrm{p}}}^{m}\left(\mu_{\mathrm{n}_{\mathrm{p}}}\right) \\
& =\sum_{m=1}^{n} \mathbf{u}^{m} \prod_{i=1}^{\mathrm{n}_{\mathrm{p}}} G_{i}^{m}\left(\mu_{i}\right) \\
& =\mathbf{U}_{\mathrm{PGD}}^{n-1}(\boldsymbol{\mu})+\mathbf{u}^{n} G_{1}^{n}\left(\mu_{1}\right) G_{2}^{n}\left(\mu_{2}\right) \ldots G_{\mathrm{n}_{\mathrm{p}}}^{n}\left(\mu_{\mathrm{n}_{\mathrm{p}}}\right)
\end{aligned}
$$

where each term, for $m=1,2, \ldots, n$ is determined by a displacement vector $\mathbf{u}^{m}$ describing the spatial mode and a set of parametric functions $G_{i}^{m}\left(\mu_{i}\right)$, for $i=1,2, \ldots, n_{\mathrm{p}}$. In the following, in order to alleviate the notation and where there is no ambiguity, the explicit dependence on $\mu_{i}$ is omitted and $G_{i}^{m}\left(\mu_{i}\right)$ is written as $G_{i}^{m}$

In order to use PGD, the input data, $\mathbf{K}(\boldsymbol{\mu})$ and $\mathbf{F}(\boldsymbol{\mu})$, has to be expressed in separated form, that is

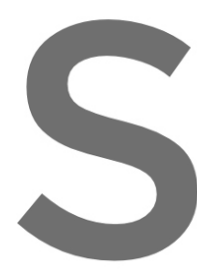

$$
\mathbb{K}(\boldsymbol{\mu})=\sum_{k=1}^{\mathrm{n}_{\mathrm{k}}} \mathbb{K}^{k} \prod_{i=1}^{\mathrm{n}_{\mathrm{p}}} B_{i}^{k}\left(\mu_{i}\right) \text { and }
$$

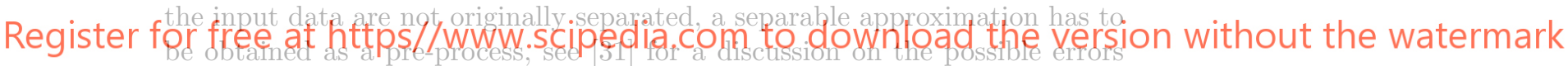
introduced in this phase.

\subsection{Sectional norms}

The PGD solver is based on the discretization and solution of the integral form of the problem presented in (3) with separable approximations. Formulating the PGD requires introducing sectional norms, that is norms along each of the independent parametric dimensions that allow measuring the modes.

For instance, the modes $G_{i}^{m} \in \mathcal{L}_{2}\left(I_{i}\right)$ describing $\mathbf{U}_{\mathrm{PGD}}$ in (5) are naturally measured with the standard norm in $\mathcal{L}_{2}\left(I_{i}\right)$, namely

$$
\left\|G_{i}^{m}\right\|^{2}=\int_{I_{i}}\left(G_{i}^{m}\right)^{2} \mathrm{~d} \mu_{i}
$$


The choice for the sectional norm affecting the space (or physical) dimension, that is the norm to measure the modal vectors $\mathbf{u}^{m} \in \mathbb{R}^{\mathbf{n}_{\mathrm{dof}}}$, is not as trivial. The obvious choice of selecting the Euclidean scalar product in $\mathbb{R}^{\mathrm{n}_{\mathrm{dof}}}$ would yield

$$
\left\|\mathbf{u}^{m}\right\|^{2}=\left[\mathbf{u}^{m}\right]^{\top} \mathbf{u}^{m} .
$$

The Euclidean norm, however, does not take into account the nature of the generalized nodal displacements (mixing displacements and rotations) and the resulting measure lacks of physical meaning. A more suitable choice is using a structural mass matrix $\mathbf{M}_{\mathbf{u}}$ with sound physical rationale that results in a norm such that

$$
\left\|\mathbf{u}^{m}\right\|^{2}=\left[\mathbf{u}^{m}\right]^{\top} \mathbf{M}_{\mathbf{u}} \mathbf{u}^{m} .
$$

Once these norms are available, it is interesting normalizing the modes, that is taking

$$
\widetilde{\mathbf{u}}^{m}=\frac{1}{\left\|\mathbf{u}^{m}\right\|} \mathbf{u}^{m} \text { and } \widetilde{G}_{i}^{m}=\frac{1}{\left\|G_{i}^{m}\right\|} G_{i}^{m} .
$$

Thus, the separated representation of $(5)$ is rewritten as

$$
\mathbf{U}_{\mathrm{PGD}}^{n}(\boldsymbol{\mu})=\sum_{m=1}^{n} \beta^{m} \widetilde{\mathbf{u}}^{m} \prod_{i=1}^{\mathrm{n}_{\mathrm{p}}} \widetilde{G}_{i}^{m},
$$

being $\beta^{m}=\left\|\mathbf{u}^{m}\right\| \prod_{i=1}^{\mathrm{n}_{\mathrm{p}}}\left\|G_{i}^{m}\right\|$ the amplitude of term $m$ of the separable sum. Note that $\beta^{m}$ provides key information on the relative importance of the different modes in the of the criteria to decide the desired accuracy. A global norm in $[\mathcal{L}(\mathcal{D})]^{\mathrm{n}_{\text {dof }}}$
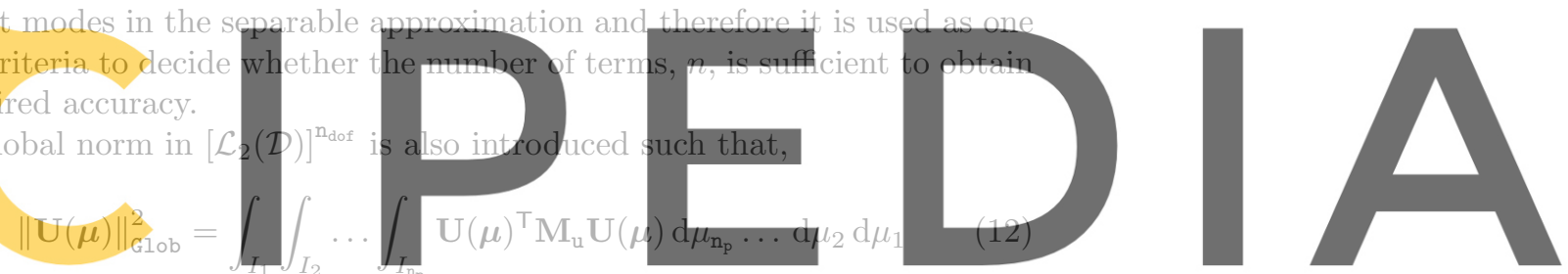

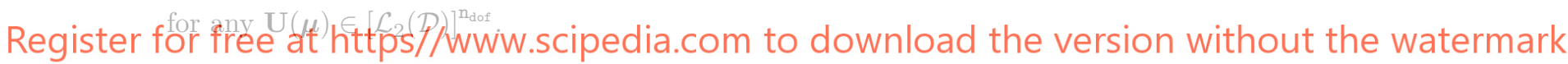

3.3 Greedy strategy and rank-one approximation

The PGD methodology aims at numerically solving (3) using an approximation of the form shown in (5). This is performed using a greedy strategy, that is computing sequentially the terms of the sum in (5). Thus, we start computing $\mathbf{U}_{\mathrm{PGD}}^{1}$, and then, when $\mathbf{U}_{\mathrm{PGD}}^{1}$ is available, compute $\mathbf{U}_{\mathrm{PGD}}^{2}$, and then $\mathbf{U}_{\mathrm{PGD}}^{3} \ldots$ Each of the steps of the greedy algorithm (that is, updating some $\mathbf{U}_{\mathrm{PGD}}^{n-1}$ into $\mathbf{U}_{\mathrm{PGD}}^{n}$ ) consists in solving a rank-one approximation problem.

Accordingly, it is assumed that $\mathbf{U}_{\mathrm{PGD}}^{n-1}$ is known and that

$$
\mathbf{U}_{\mathrm{PGD}}^{n}=\mathbf{U}_{\mathrm{PGD}}^{n-1}+\mathbf{u} \prod_{i=1}^{\mathrm{n}_{\mathrm{p}}} G_{i},
$$


where the superscript $n$ in $\mathbf{u}^{n}$ and $G_{i}^{n}$ is here omitted to simplify the notation.

The problem consists now in finding $\mathbf{u}, G_{1}, G_{2}, \ldots, G_{\mathrm{n}_{\mathrm{p}}}$ such that $\mathbf{U}_{\mathrm{PGD}}^{n}$ fulfills (3). The complete unknown function $\mathbf{u} \prod_{i=1}^{\mathrm{n}_{\mathrm{p}}} G_{i}$ is said to be of rank one because it is build as the product of sectional functions. The problem at hand is nonlinear (because the unknown functions are multiplying each other) but with a number of degrees of freedom much lower than the original one. Recall that the discrete version of the full linear problem has a number of degrees of freedom $\mathrm{n}_{\mathrm{Full}}$ given in (4). Instead, with the PGD strategy, each rank-one approximation problem is nonlinear but with a number of degrees of freedom, $\mathrm{n}_{\text {Rankone }}$ given by:

$$
\mathrm{n}_{\text {RankOne }}=\mathrm{n}_{\text {dof }}+\sum_{i=1}^{\mathrm{n}_{\mathrm{p}}} \mathrm{n}_{\mathrm{d}, i} .
$$

Typically, due to the additive nature of $\mathrm{n}_{\text {Rank0ne }}$ in terms of the sectional dimensions, $n_{\text {Rankone }} \ll n_{\text {Full }}$ holds and therefore the reduction of the dimension of the problem is well worth the difficulties associated with the newly acquired nonlinear character.

Due to the incremental character of (13), the expression of the residual (2) is rewritten as

$$
\mathbf{R}\left(\mathbf{U}_{\mathrm{PGD}}^{n}\right)=\mathbf{R}\left(\mathbf{U}_{\mathrm{PGD}}^{n-1}\right)-\mathbf{K}(\boldsymbol{\mu}) \mathbf{u} \prod_{i=1}^{\mathrm{n}_{\mathrm{p}}} G_{i},
$$

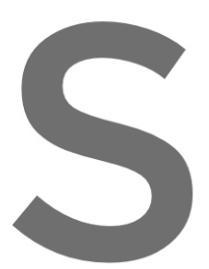

which, using (6), becomes
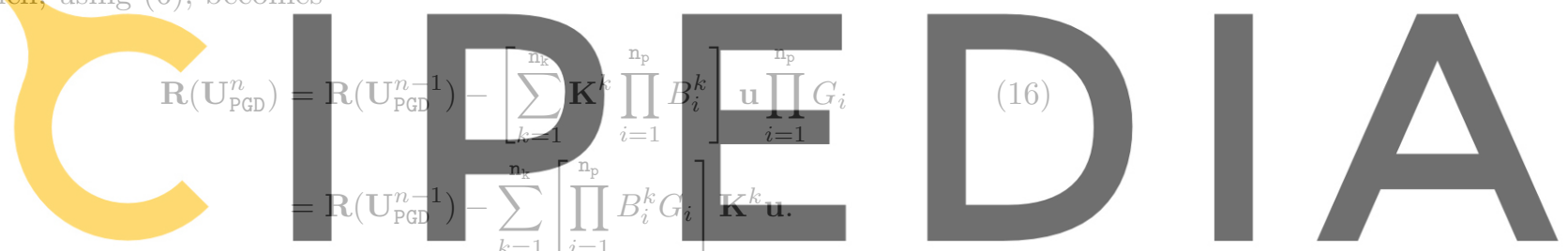

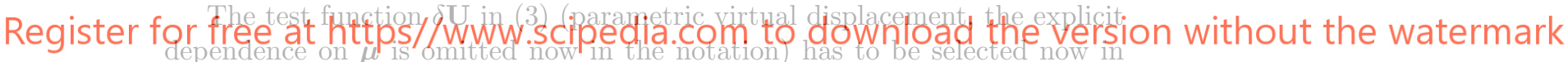 \\ accordance with the unknown of the rank-one problem consisting in introduc-}

ing in (3) the residual as defined in (15) or (16). This test function is taken as a variation of the actual unknown $\mathbf{u} \prod_{i=1}^{\mathrm{n}_{\mathrm{p}}} G_{i}$ and yields

$$
\delta \mathbf{U}=\delta \mathbf{u} \prod_{i=1}^{\mathrm{n}_{\mathrm{p}}} G_{i}+\mathbf{u} \sum_{i=1}^{\mathrm{n}_{\mathrm{p}}} \delta G_{i} \prod_{\substack{j=1 \\ j \neq i}}^{\mathrm{n}_{\mathrm{p}}} G_{j} .
$$

That is, instead of taking an arbitrary $\delta \mathbf{U} \in\left[\mathcal{L}_{2}(\mathcal{D})\right]^{\mathrm{n}_{\mathrm{dof}}}$ as in $(3)$, one must take arbitrary $\delta \mathbf{u} \in \mathbb{R}^{\mathrm{n}_{\text {dof }}}$ and $\delta G_{i} \in \mathcal{L}_{2}\left(I_{i}\right)$, for $i=1,2, \ldots, \mathrm{n}_{\mathrm{p}}$. Then, in the numerical version, the finite-dimensional sectional spaces $V_{i}$ replace $\mathcal{L}_{2}\left(I_{i}\right)$.

The numerical strategy to deal with the nonlinearity of this problem is based on the alternated directions idea [3]. 


\subsection{Alternated directions solver}

The alternated directions strategy to solve the rank-one nonlinear problem consists in successively solving for each one of the unknowns assuming that the rest of them are known.

\subsubsection{Computing $\mathbf{u}$}

Thus, the first step consists in, assuming $G_{i}$ known, for $i=1,2, \ldots, \mathrm{n}_{\mathrm{p}}$, compute $\mathbf{u}$ such that $\mathbf{R}\left(\mathbf{U}_{\mathrm{PGD}}^{n}\right)$ as defined in (16) satisfies (3). Since $G_{i}$ is known, $\delta G_{i}$ is taken to be zero, for $i=1,2, \ldots, \mathrm{n}_{\mathrm{p}}$, and $\delta \mathbf{U}$ in (17) reduces to

$$
\delta \mathbf{U}=\delta \mathbf{u} \prod_{i=1}^{\mathrm{n}_{\mathrm{p}}} G_{i} .
$$

Thus, in this case (3) becomes

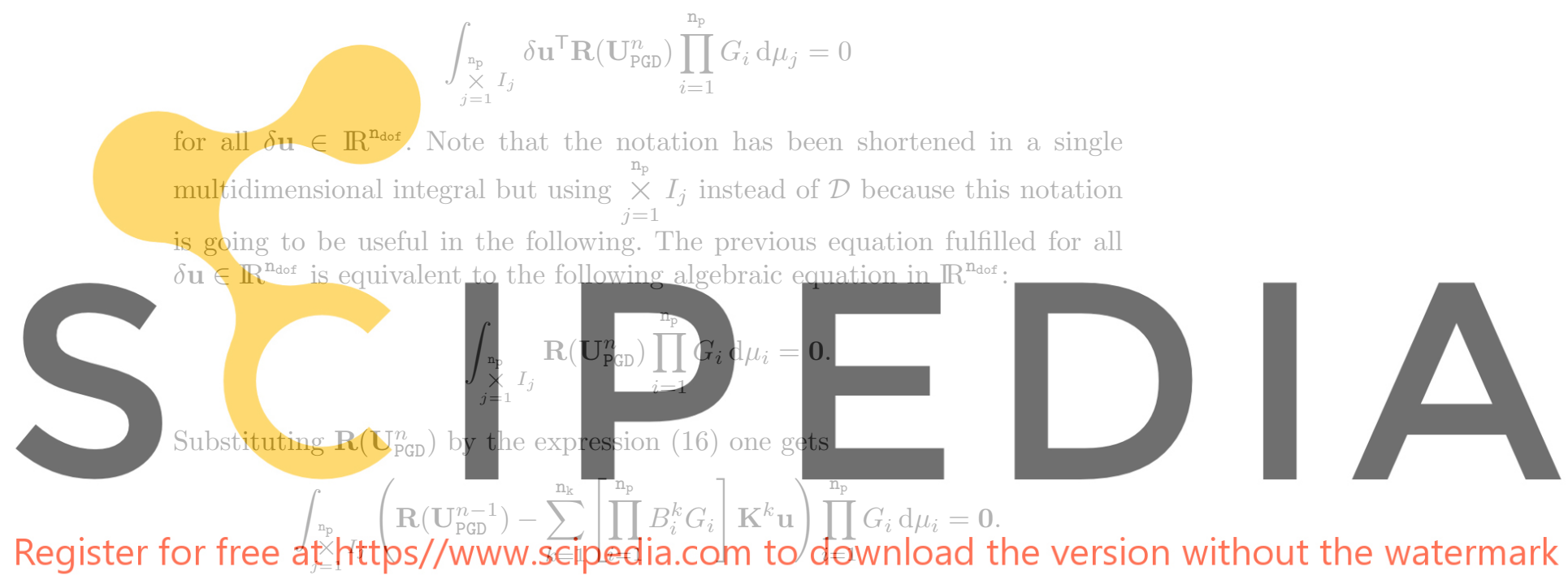

Using the expressions for $\mathrm{U}(\mu), \mathbb{K}(\mu)$ and $\mathbf{F}(\mu)$ in (5), (6) and (7), respec-

tively, and the definition of the residual in (2) the resuiting equation reads

$$
\begin{aligned}
{[\sum_{k=1}^{\mathrm{n}_{\mathrm{k}}} \mathbf{K}^{k} \underbrace{\prod_{i=1}^{\mathrm{n}_{\mathrm{p}}} \int_{I_{i}} B_{i}^{k}\left(G_{i}\right)^{2} \mathrm{~d} \mu_{i}}_{c^{k}}] \mathbf{u}=} & \sum_{\ell=1}^{\mathrm{n}_{\mathrm{f}}} \mathbf{f}^{\ell} \overbrace{i=1}^{\overbrace{\mathrm{n}_{\mathrm{p}}} \int_{I_{i}} S_{i}^{\ell} G_{i} \mathrm{~d} \mu_{i}} \\
& -\sum_{m=1}^{\hat{c}^{\ell}}[\sum_{k=1}^{\mathrm{n}_{\mathrm{k}}} \mathbf{K}^{k} \underbrace{\mathrm{n}_{i}}_{c_{i=1}^{\mathrm{n}_{\mathrm{p}}} \int_{I_{i}} B_{i}^{k} G_{i}^{m} G_{i} \mathrm{~d} \mu_{i}}] \mathbf{u}^{m} .
\end{aligned}
$$


That is, a linear system of equations for $\mathbf{u}$ :

$$
\left[\sum_{k=1}^{\mathrm{n}_{\mathrm{k}}} \mathbf{K}^{k} c^{k}\right] \mathbf{u}=\sum_{\ell=1}^{\mathrm{n}_{\mathrm{f}}} \mathbf{f}^{\ell} \hat{c}^{\ell}-\sum_{m=1}^{n-1}\left[\sum_{k=1}^{\mathrm{n}_{\mathrm{k}}} \mathbf{K}^{k} c^{k, m}\right] \mathbf{u}^{m}
$$

where scalars $c^{k}, \hat{c}^{\ell}$ and $c^{k, m}$ are computable terms defined by:

$$
\begin{aligned}
c^{k} & :=\prod_{i=1}^{\mathrm{n}_{\mathrm{p}}} \int_{I_{i}} B_{i}^{k}\left(G_{i}\right)^{2} \mathrm{~d} \mu_{i}, \\
\hat{c}^{\ell} & :=\prod_{i=1}^{\mathrm{n}_{\mathrm{p}}} \int_{I_{i}} S_{i}^{\ell} G_{i} \mathrm{~d} \mu_{i} \text { and } \\
c^{k, m} & :=\prod_{i=1}^{\mathrm{n}_{\mathrm{p}}} \int_{I_{i}} B_{i}^{k} G_{i}^{m} G_{i} \mathrm{~d} \mu_{i} .
\end{aligned}
$$

3.4.2 Computing $G_{i}$, for $i=1,2, \ldots, n_{p}$

The subsequent steps consist in computing one parametric mode, say $G_{i}$, assuming that $\mathbf{u}$ and the rest of the modes $G_{j}$, for $j=1,2, \ldots, \mathrm{n}_{\mathrm{p}}$ and $j \neq i$, are known and therefore the corresponding variations $\delta G_{j}$ and $\delta \mathbf{u}$ are taken to be zero. Thus, the variation $\delta \mathbf{U}$ in (17) becomes
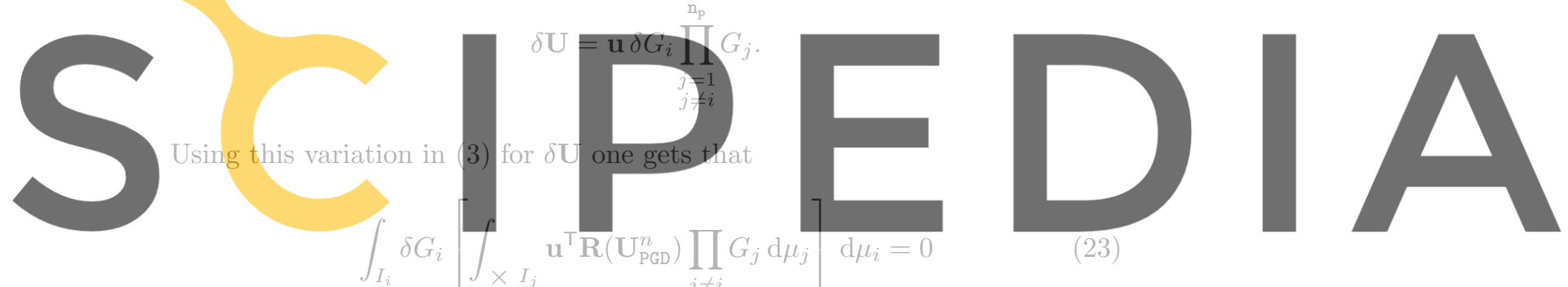

Register for free at https//www.scipedia.com to download the version without the watermark

must hold for all $\delta G_{i} \in \mathcal{L}_{2}\left(I_{i}\right)$. In order to ease the reading, the range of $j$ is

omitted, emphasizing only the fact that $j \neq i$. Note that (23) is an equation

for the unknown function $G_{i}(\cdot)$ that appears explicitly in $\mathbf{R}\left(\mathbf{U}_{\mathrm{PGD}}^{n}\right)$, see (15).

Indeed, the integral equation (23) results in the following equation for $G_{i}(\cdot)$

$$
\int_{\substack{\times \neq i \\ j \neq i}} \mathbf{u}^{\top}\left[\mathbf{R}\left(\mathbf{U}_{\mathrm{PGD}}^{n-1}\right)-G_{i} \mathbf{K}(\boldsymbol{\mu}) \mathbf{u} \prod_{j \neq i} G_{j}\right] \prod_{j \neq i} G_{j} \mathrm{~d} \mu_{j}=0 .
$$

That is

$$
G_{i}\left[\int_{\substack{\times \neq i \\ j \neq i}} \mathbf{u}^{\top} \mathbf{K}(\boldsymbol{\mu}) \mathbf{u} \prod_{j \neq i}\left(G_{j}\right)^{2} \mathrm{~d} \mu_{j}\right]=\int_{\underset{j \neq i}{\times} I_{j}} \mathbf{u}^{\top} \mathbf{R}\left(\mathbf{U}_{\mathrm{PGD}}^{n-1}\right) \prod_{j \neq i} G_{j} \mathrm{~d} \mu_{j},
$$


where both the term in square brackets in the left-hand side and the righthand side of (25) are computable functions in $I_{i}$. The expression for these functions is readily determined using the forms of the data given in (5), (6) and (7). The resulting expressions read

$$
G_{i}(\cdot)=\frac{\sum_{\ell=1}^{\mathrm{n}_{\mathrm{f}}}\left(\mathbf{u}^{\top} \mathbf{f}^{\ell}\right) \hat{d}_{i}^{\ell}(\cdot)-\sum_{m=1}^{n-1} \sum_{k=1}^{\mathrm{n}_{\mathrm{k}}}\left(\mathbf{u}^{\top} \mathbf{K}^{k} \mathbf{u}^{m}\right) d_{i}^{k, m}(\cdot)}{\sum_{k=1}^{\mathrm{n}_{\mathrm{k}}}\left(\mathbf{u}^{\top} \mathbf{K}^{k} \mathbf{u}\right) d_{i}^{k}(\cdot)},
$$

where the computable functions $d_{i}^{k}(\cdot), \hat{d}_{i}^{\ell}(\cdot)$ and $d_{i}^{k, m}(\cdot)$ taking values in $I_{i}$ are defined by

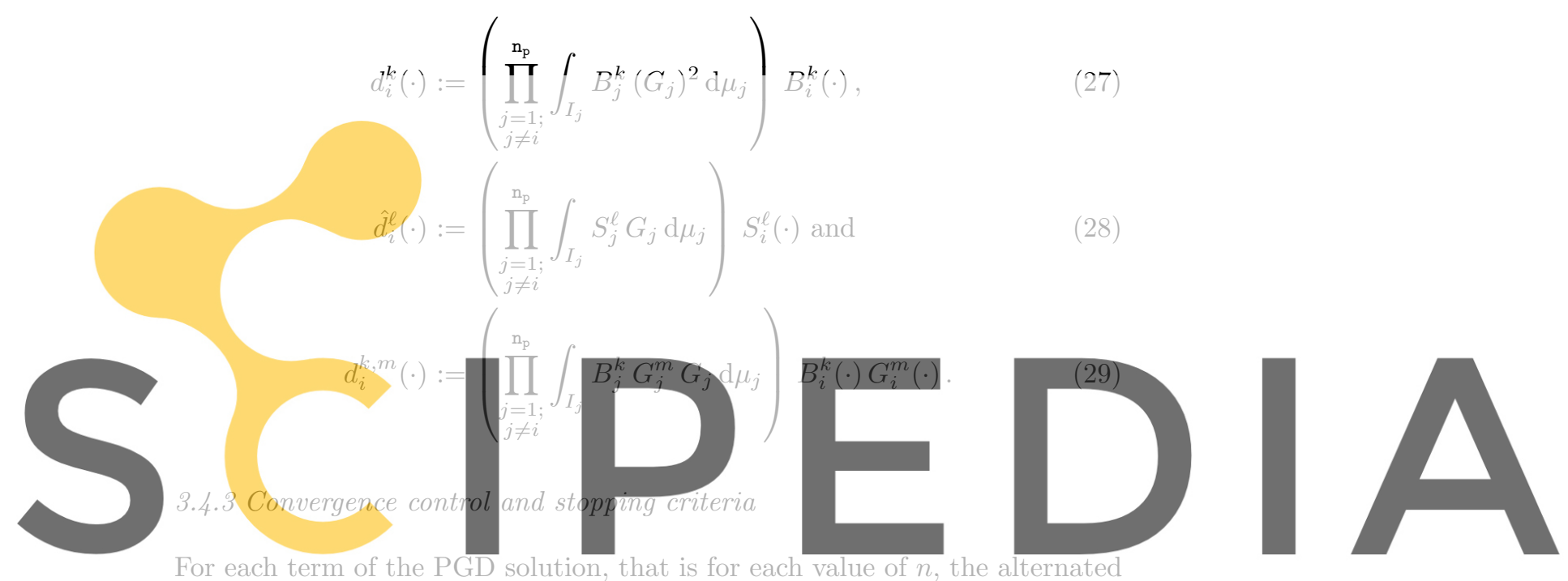

For each term of the PGD solution, that is for each value of $n$, the alternated

directions iterations are expected to converge to the best rank-one approxi-.

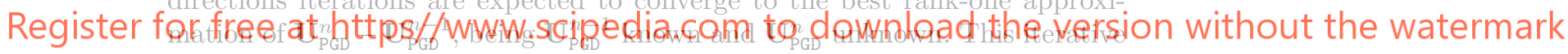

algorithm (see Appendix A) requires a stopping criterion to decide whether

the current iteration is acceptable or not. The stopping criterion is based on

the stationarity of the solution: the iteration is validated if the modification

from the previous iteration is small enough. Thus, assume that $\mathbf{u}$ and $G_{i}$, for $i=1,2, \ldots, \mathrm{n}_{\mathrm{p}}$ characterize the previous iteration and that after the alternated directions loop the new approximation is given by $\mathbf{u}^{\text {new }}$ and $G_{i}^{\text {new }}$, for $i=1,2, \ldots, \mathrm{n}_{\mathrm{p}}$. The stopping criteria is expressed in terms of the difference between the two successive iterations, measured with the norms introduced in Section 3.2 .

In particular, a typical convergence criterion is to continue iterating while

$$
\left\|\mathbf{u}^{\text {new }} \prod_{i=1}^{\mathrm{n}_{\mathrm{p}}} G_{i}^{\text {new }}-\mathbf{u} \prod_{i=1}^{\mathrm{n}_{\mathrm{p}}} G_{i}\right\|_{\mathrm{Glob}}>\eta_{\mathrm{tol}}\left\|\mathbf{u}^{\text {new }} \prod_{i=1}^{\mathrm{n}_{\mathrm{p}}} G_{i}^{\text {new }}\right\|_{\text {Glob }},
$$


where $\|\cdot\|_{\text {Glob }}$ is introduced in (12) and $\eta_{\text {tol }}$ is a user-prescribed tolerance.

The computation of these norms is simplified by using the normalized modes, $\widetilde{\mathbf{u}}$ and $\widetilde{G}_{i}$, introduced in (10) and the amplitude $\beta$. Namely,

$$
\begin{aligned}
\left\|\mathbf{u}^{\text {new }} \prod_{i=1}^{\mathrm{n}_{\mathrm{p}}} G_{i}^{\text {new }}-\mathbf{u} \prod_{i=1}^{\mathrm{n}_{\mathrm{p}}} G_{i}\right\|_{\mathrm{Glob}}^{2}= & \left\|\mathbf{u}^{\text {new }} \prod_{i=1}^{\mathrm{n}_{\mathrm{p}}} G_{i}^{\text {new }}\right\|_{\text {Glob }}^{2}+\left\|\mathbf{u} \prod_{i=1}^{\mathrm{n}_{\mathrm{p}}} G_{i}\right\|_{\mathrm{Glob}}^{2} \\
& -2\left(\mathbf{u}^{\text {new }}, \mathbf{u}\right) \prod_{i=1}^{\mathrm{n}_{\mathrm{p}}}\left(G_{i}^{\text {new }}, G_{i}\right) \\
= & \left(\beta^{\text {new }}\right)^{2}+\beta^{2} \\
& -2 \beta^{\text {new }} \beta\left(\widetilde{\mathbf{u}}^{\top} \mathbf{M}_{\mathbf{u}} \widetilde{\mathbf{u}}^{\text {new }}\right) \prod_{i=1}^{\mathrm{n}_{\mathrm{p}}} \int_{I_{i}} \widetilde{G}_{i}^{\text {new }} \widetilde{G}_{i} \mathrm{~d} \mu_{i},
\end{aligned}
$$

where all the sectional scalar products are denoted in a unified fashion by the bilinear operator $(\cdot, \cdot)$. Note that if the new iteration coincides with the previous one, this expression vanishes because the amplitudes are equal and the scalar product of the identical and normalized modes is equal to one.

In Appendix A the algorithm of the alternated directions solver is detailed, see Algorithm 1.

\subsection{PGD compression}

The $n$ terms of PGD solution $\mathbf{U}_{\mathrm{PGD}}^{n}$ may contain redundant information. This is associated with the greedy strategy employed to compute the successive terms, with no enforcement of any orthogonality between the successive modes. Thus, the number of terms in an optimal separable approximation required to achieve the same level of accuracy as in $\mathbf{U}_{\mathrm{PGD}}^{n}$ is often much lower than $n$. This can be checked a posteriori in the 2D case, where the Singular Value Decomposition (SVD) provides an optimal separation, with the least number of terms. For higher dimensions, the problem still holds but there is no optimal solution to compare with (the High Order SVD, or HO-SVD, is no longer optimal).

In order to mitigate the effect of this phenomenon, a common practice in the PGD codes is to implement the so-called PGD compression, see the appendix in [22] where it is referred also as HO-PGD. This compression consists in a least-squares projection of $\mathbf{U}_{\mathrm{PGD}}^{n}$ into the same approximation space, computed with the very same PGD strategy, that is combining a greedy algorithm for the terms and an alternated directions scheme for the modes.

In the context of the parametric structural problem (1), the PGD compression is formulated as follows. Let $\mathbf{U}_{\mathrm{PGD}}^{n}$ be the raw PGD solution with $n$ terms. Instead of solving the original equation (1) or its integral counterpart (3), now the objective is obtaining $\mathbf{U}_{\text {com }}$ minimizing the least-squares functional $\mathcal{J}$ defined as

$$
\mathcal{J}\left(\mathbf{U}_{\mathrm{com}}\right)=\left\|\mathbf{U}_{\mathrm{com}}-\mathbf{U}_{\mathrm{PGD}}^{n}\right\|_{\mathrm{Glob}}^{2}
$$


More precisely, the aim is at computing a separated approximation $\mathbf{U}_{\mathrm{com}}^{\widehat{n}}$ with $\widehat{n}$ terms expressed as

$$
\mathbf{U}_{\text {com }}^{\widehat{n}}=\sum_{\widehat{m}=1}^{\widehat{n}} \widehat{\mathbf{u}}^{\widehat{m}} \prod_{i}^{\mathrm{n}_{\mathrm{p}}} \widehat{G}_{i}^{\widehat{m}}
$$

using a PGD approach to minimize (32) with the expectation of getting a sufficiently accurate approximation to $\mathbf{U}_{\mathrm{PGD}}^{n}$ with $\widehat{n} \ll n$.

Again, the main idea of the PGD strategy is formulating the problem of finding a rank-one approximation. Thus, let us briefly describe how to compute the first term of $\mathbf{U}_{\text {com }}^{\widehat{n}}$ taking

$$
\mathbf{U}_{\mathrm{com}}=\widehat{\mathbf{u}} \prod_{i}^{\mathrm{n}_{\mathrm{p}}} \widehat{G}_{i} .
$$

Following the expansion in (31), the expression for $\mathcal{J}\left(\mathbf{U}_{\text {com }}\right)$ is reduced to its dependence on the unknowns $\widehat{\mathbf{u}}, \widehat{G}_{1}, \ldots, \widehat{G}_{\mathrm{n}_{\mathrm{p}}}$ and reads

$$
\begin{aligned}
\mathcal{J}\left(\widehat{\mathbf{u}}, \widehat{G}_{1}, \ldots, \widehat{G}_{\mathrm{n}_{\mathrm{p}}}\right)= & \left\|\widehat{\mathbf{u}} \prod_{i=1}^{\mathrm{n}_{\mathrm{p}}} \widehat{G}_{i}\right\|_{G 1 \mathrm{ob}}^{2}+\left\|\sum_{m=1}^{n} \mathbf{u}^{m} \prod_{i=1}^{\mathrm{n}_{\mathrm{p}}} G_{i}^{m}\right\|_{\text {Glob }}^{2} \\
& -2 \sum_{m=1}^{n}\left(\widehat{\mathbf{u}}, \mathbf{u}^{m}\right) \prod_{i=1}^{\mathrm{n}_{\mathrm{p}}}\left(\widehat{G}_{i}, G_{i}^{m}\right) .
\end{aligned}
$$

Again the alternated direction scheme is used here. The first step assumes that $\widehat{G}_{1}, \ldots, \widehat{G}_{\mathrm{n}_{\mathrm{p}}}$ are known to compute $\widehat{\mathbf{u}}$. Note that the previous functional is quadratic for $\widehat{\mathbf{u}}$ and therefore the sectional minimization problem results in solving a linear system of equations for $\widehat{\mathbf{u}}$. Indeed, for given values of $\widehat{G}_{1}, \ldots, \widehat{G}_{\mathrm{n}_{\mathrm{p}}}$, the functional dependence on the remaining unknown reads

$$
\begin{aligned}
\mathcal{J}(\widehat{\mathbf{u}})= & \left(\widehat{\mathbf{u}}^{\top} \mathbf{M}_{\mathbf{u}} \widehat{\mathbf{u}}\right) \overbrace{\prod_{i=1}^{\mathrm{n}_{\mathrm{p}}}\left(\widehat{G}_{i}, \widehat{G}_{i}\right)}^{\lambda}+\left\|\sum_{m=1}^{n} \mathbf{u}^{m} \prod_{i=1}^{\mathrm{n}_{\mathrm{p}}} G_{i}^{m}\right\|_{\text {Glob }}^{2} \\
& -2 \sum_{m=1}^{n}\left(\widehat{\mathbf{u}}^{\top} \mathbf{M}_{\mathbf{u}} \mathbf{u}^{m}\right) \underbrace{\underbrace{m}}_{\prod_{i=1}^{\mathrm{n}_{\mathrm{p}}}\left(\widehat{G}_{i}, G_{i}^{m}\right)}
\end{aligned}
$$

and minimizing $\mathcal{J}(\widehat{\mathbf{u}})$ requires solving $\frac{\partial \mathcal{J}(\widehat{\mathbf{u}})}{\partial \widehat{\mathbf{u}}}=0$, that is

$$
\mathbf{M}_{\mathbf{u}} \widehat{\mathbf{u}}=\frac{1}{\lambda} \sum_{m=1}^{n} \gamma^{m} \mathbf{M}_{\mathbf{u}} \mathbf{u}^{m} \Longrightarrow \widehat{\mathbf{u}}=\frac{1}{\lambda} \sum_{m=1}^{n} \gamma^{m} \mathbf{u}^{m}
$$

Note that matrix $\mathbf{M}_{\mathbf{u}}$ cancels in both sides and the solution of the linear system of equations is provided by an explicit expression. 
The iteration to compute modal function $\widehat{G}_{i}$, provided that $\widehat{\mathbf{u}}$ and $\widehat{G}_{j}$ are available for $j \neq i$, leads also to a simple equation. The sectional functional to be minimized reads

$$
\begin{aligned}
\mathcal{J}\left(\widehat{G}_{i}\right)= & \overbrace{\left[\left(\widehat{\mathbf{u}}^{\top} \mathbf{M}_{\mathbf{u}} \widehat{\mathbf{u}}\right) \prod_{j \neq i}\left(\widehat{G}_{j}, \widehat{G}_{j}\right)\right]}^{\eta_{i}}\left(\widehat{G}_{i}, \widehat{G}_{i}\right)+\left\|\sum_{m=1}^{n} \mathbf{u}^{m} \prod_{i=1}^{\mathrm{n}_{\mathrm{p}}} G_{i}^{m}\right\|_{\mathrm{Glob}}^{2} \\
& -2 \sum_{m=1}^{n} \underbrace{\left(\widehat{\mathbf{u}}^{\mathrm{T}} \mathbf{M}_{\mathbf{u}} \mathbf{u}^{m}\right) \prod_{j \neq i}\left(\widehat{G}_{j}, G_{j}^{m}\right)}_{\xi_{i}^{m}}\left(\widehat{G}_{i}, G_{i}^{m}\right),
\end{aligned}
$$

which results in

$$
\widehat{G}_{i}(\cdot)=\frac{1}{\eta_{i}} \sum_{m=1}^{n} \xi_{i}^{m} G_{i}^{m}(\cdot) .
$$

Note that expressions (34) and (35) provide a very simple solver for each of the alternated directions iteration. In the case of the subsequent terms of $\mathbf{U}_{\text {com }}^{\widehat{n}}$, that is for $\widehat{n}>1$, the expressions are very similar because one has to replace only $\mathbf{U}_{\mathrm{PGD}}^{n}$ by $\mathbf{U}_{\mathrm{PGD}}^{n}-\mathbf{U}_{\mathrm{com}}^{\widehat{n}-1}$. Therefore, the expressions (34) and (35) are not significantly different, just that the sums take $n+\widehat{n}-1$ terms instead of $n$.

The stopping criteria in this case are identical to those presented in Section 3.4.3. Again, in the rank-one approximation, the only check that has to be performed is that the iterations have reached a stationary configuration. In fact, the same expression as in equation (31) can be straightforwardly used here taking $\widehat{\mathbf{u}}$ instead of $\mathbf{u}$ and $\widehat{G}_{i}$ instead of $G_{i}$. 
4 Numerical examples: application to structured auxetic patterns

The generalization of PGD to structural problems is used to model a parametric architectured material which is expected to exhibit auxetic properties (that is, negative Poisson ratios). The inverted honeycomb shaped cell is adopted here as a mechanism to generate an auxetic behavior, as introduced in [1]. The geometry of the structure to be analyzed is shown in Figure 1 and the corresponding unit-cell and its parameterization are illustrated in Figure 2. The unit-cell is formed by 8 segments (numbered with lowercase roman numbers, $i, i i, \ldots$, vii, viii) modelled by beam elements and the actual shape of the cell is characterized by the following parameters:

- $a$ : the length of the obliquely oriented beam elements $i i, i v, v i$ and viii.

- $b$ : the length of horizontal beam elements $i i i$ and vii (this parameter is set to one, $b=1$, in the following examples).

$-t$ : the thickness of the elements $i i i$ and vii, being $2 t$ in the others.

- $\alpha$ : the angle of the elements $i i, i v, v i$ and viii with respect to the horizontal.

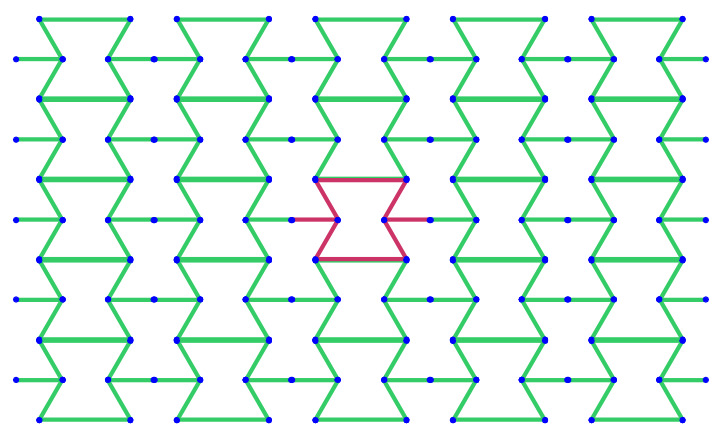

Fig. 1: 2D Architectured material formed by a periodic repetition of a unit-cell, marked in red. 


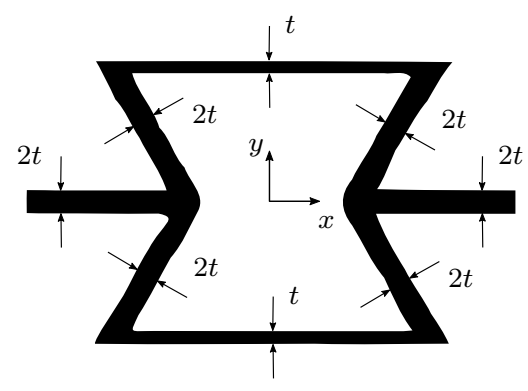

(a) Cell characterization

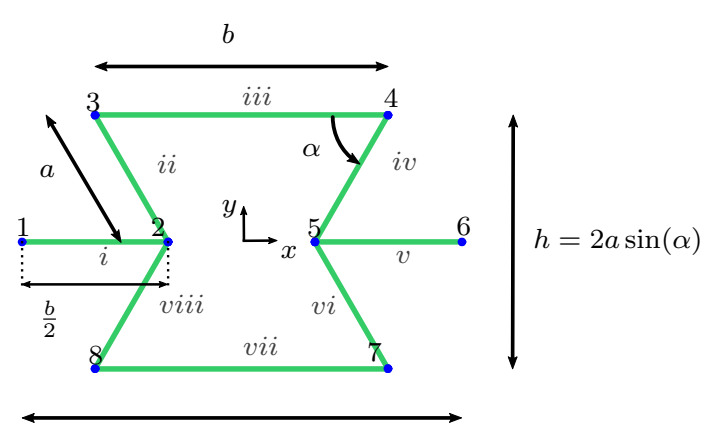

$w=2(b-a \cos (\alpha))$

(b) Beam discretization

Fig. 2: Inverted honeycomb shaped unit-cell with its parameterization and numbering of the nodes and elements.

Thus, the $\mathrm{n}_{\mathrm{p}}=3$ free parameters are $t, a$ and $\alpha$, that is;

$$
\boldsymbol{\mu}=\left[\begin{array}{lll}
\mu_{1} & \mu_{2} & \mu_{3}
\end{array}\right]^{\top}=\left[\begin{array}{lll}
t & a & \alpha
\end{array}\right]^{\top} .
$$

The width of the cell, $w$ in Figure 2 has to be larger than $b$ and therefore the restriction $\cos \alpha<b / 2 a$ must hold.

For our parametric analyses, the parameters were chosen to range in the following intervals

$$
t \in I_{1}=\left[\frac{1}{200}, \frac{1}{20}\right] ; a \in I_{2}=[0.3,0.7] \text { and } \alpha \in I_{3}=\left[\frac{\pi}{4}, \frac{3 \pi}{4}\right] .
$$

That is, the solution $\mathbf{U}_{\mathrm{PGD}}^{n}(\cdot)$ takes values in $\mathcal{D}=I_{1} \times I_{2} \times I_{3}$, where $\boldsymbol{\mu}$ ranges. The corresponding intervals are uniformly discretized such that the spaces $V_{1}$, $V_{2}$ and $V_{3}$ have dimensions $\mathrm{n}_{\mathrm{d}, 1}=\mathrm{n}_{\mathrm{d}, 2}=100$ and $\mathrm{n}_{\mathrm{d}, 3}=500$.

The loads in all the examples are enforced via prescribed displacements (no tractions are applied on the boundary). In the analysis of the unit-cell, this includes periodicity constraints. The expressions for the parametric dependence of stiffness and mass matrices can be easily derived from standard Bernoulli beam elements. Recall that the free parameters describing the lattice structure are $t, a$ and $\alpha$. The other parameters of the beams ( $b$, Young's modulus, depth of the rectangular cross-section) are taken to be equal to one. The non-homogeneous Dirichlet boundary conditions (nonzero prescribed displacements) are enforced in the first PGD mode, thus homogeneous essential conditions are enforced in the subsequent modes.

The tolerance $\eta_{\mathrm{tol}}$ in (30) to control the convergence of the alternated directions iteration is set to $10^{-6}$, see schematic implementation in Algorithms 1 and 2 of Appendix A. To control the number of terms in the PGD expansion, the criterion selected is based on the reduction of the amplitudes $\beta^{m}, m=$ 


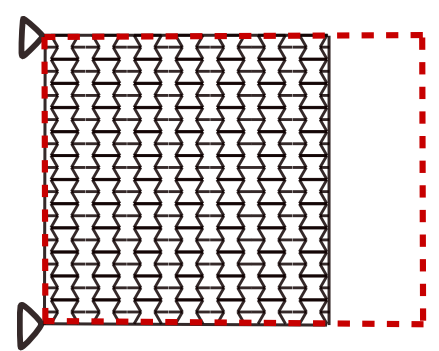

(a) Load Case $1=X X$

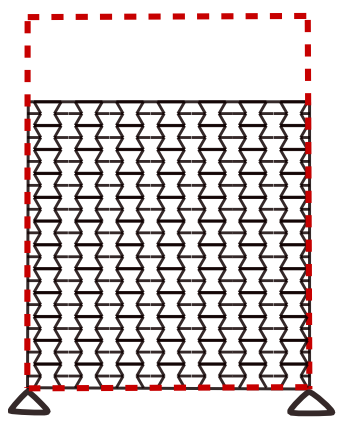

(b) Load Case $2=Y Y$

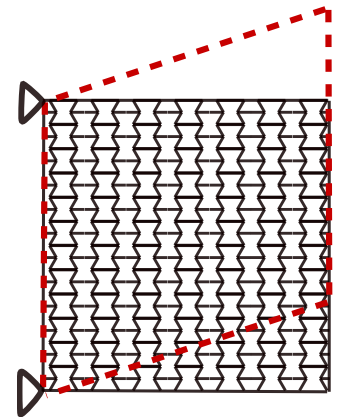

(c) Load Case $3=X Y$

Fig. 3: Prescribed load cases for the material structure at macro-scale.

$1,2, \ldots, n$. Namely, the process is stopped at term number $n$ if

$$
\frac{\beta^{n}}{\max _{m=1, \ldots, n}\left(\beta^{m}\right)}<10^{-3} .
$$

These considerations are valid for the three examples presented in the following: 1) the full parametric solution of the unit-cell for the three loading cases allowing to build up an homogenized bulk mode (Section 4.1), 2) the solution of the global parametric problem (Section 4.2) and 3) the global solution of a non fully periodic structure (Section 4.3).

\subsection{Unit-cell problem using homogenization}

The goal here is to exploit the generalized solution of the parametric unitcell to extract the effective mechanical properties at the macro-scale. As it is standard in the homogenization practice, see $[4,20]$ and references therein, the unit-cell has to be subjected to different periodic loading conditions. In this case three loading cases are considered as described in Figure 3: the three solutions are denoted $\mathbf{U}^{1} \equiv \mathbf{U}^{X X}, \mathbf{U}^{2} \equiv \mathbf{U}^{Y Y}$ and $\mathbf{U}^{3} \equiv \mathbf{U}^{X Y}$. Analyzing the unit-cell model (Figure 2) allows computing the material effective mechanical properties through homogenization theory, which describes how these loading conditions, applied to a material structure at macro-scale, should be applied equivalently to the unit-cell by using periodicity boundary conditions at microscale. For further details on homogenization, we suggest the readers the works of $[4,20]$ and references therein. Once the three solutions are available, the effective constitutive Hooke matrix for the homogenized material reads

$$
\mathbf{C}^{\text {eff }}=\left[\begin{array}{lll}
C_{11}^{\text {eff }} & C_{12}^{\text {eff }} & C_{13}^{\text {eff }} \\
C_{21}^{\text {eff }} & C_{22}^{\text {eff }} & C_{23}^{\text {eff }} \\
C_{31}^{\text {eff }} & C_{32}^{\text {eff }} & C_{33}^{\text {eff }}
\end{array}\right]
$$


where

$$
C_{I J}^{\text {eff }}(\boldsymbol{\mu})=\frac{1}{w h}\left(\mathbf{U}^{I}(\boldsymbol{\mu})\right)^{\top} \mathbf{K}(\boldsymbol{\mu}) \mathbf{U}^{J}(\boldsymbol{\mu}), \text { for } I, J=1,2,3
$$

It is worth noting that $w h$ stands for the area occupied by the cell. Note that both left and right hand sides of equation (37) have physical units of stiffness. On the left hand side, there are components of the $2 \mathrm{D}$ constitutive matrix, whereas on the right hand side we have units of energy divided by area. This corresponds to units of stiffness as well, assuming plane elasticity.

In each of the three loading cases, the results displayed are the following:

- the evolution of the modal amplitudes $\beta^{m}, m=1,2, \ldots, n$ with and without PGD compression, see Figures 4, 7 and 10

- the shape of the first modal displacements $\mathbf{u}^{1}$ and $\mathbf{u}^{2}$, see Figures 5, 8 and 11

- the normalized parametric functions, $\widetilde{G}_{i}^{m}$, for all the modes with PGD compression. In order to provide an idea of the modal amplitude, each function $\widetilde{G}_{i}^{m}$ is weighted by $\left(\beta^{m}\right)^{\frac{1}{\mathrm{n}_{\mathrm{p}}}}, \mathrm{n}_{\mathrm{p}}=3$, see Figures 6,9 and 12 .

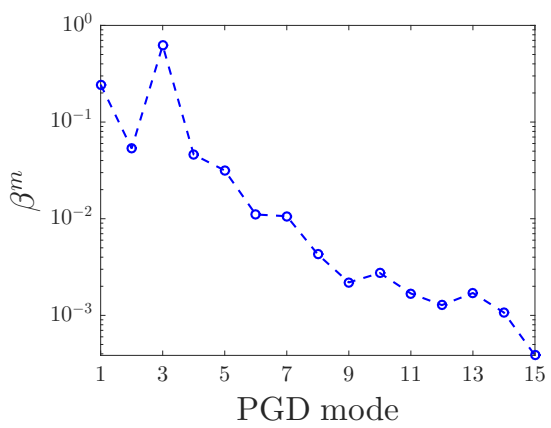

(a) No PGD compression

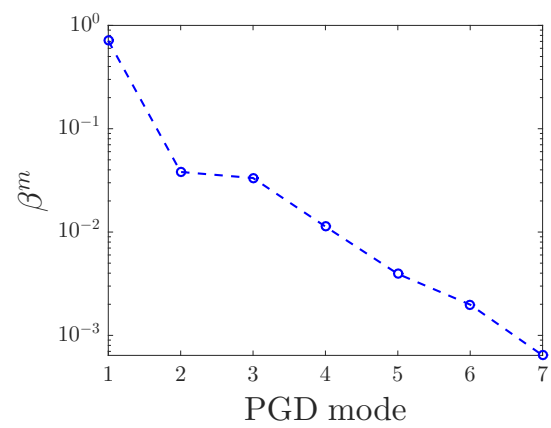

(b) With PGD compression

Fig. 4: Unit-cell: load case $X X$, evolution of modal amplitudes $\beta^{m}, m=$ $1,2, \ldots, n$. 


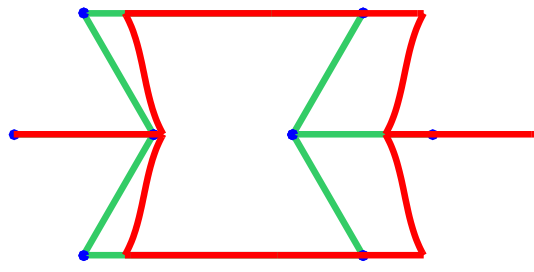

(a) Scaled $\widetilde{\mathbf{u}}^{1}$

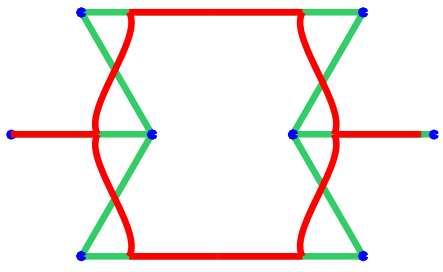

(b) Scaled $\widetilde{\mathbf{u}}^{2}$

Fig. 5: Unit-cell: load case $X X$, shape of the first modal vectors with PGD compression (in red).

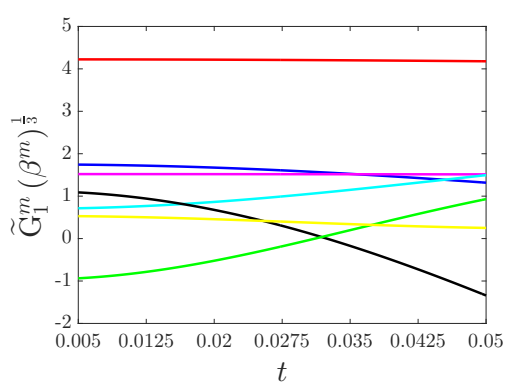

(a) Weighted $\widetilde{\mathrm{G}}_{1}^{m}$

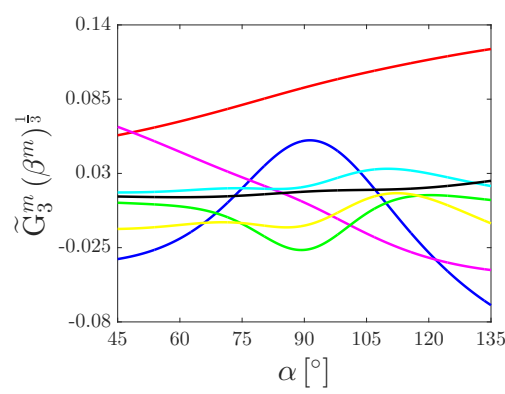

(c) Weighted $\widetilde{\mathrm{G}}_{3}^{m}$

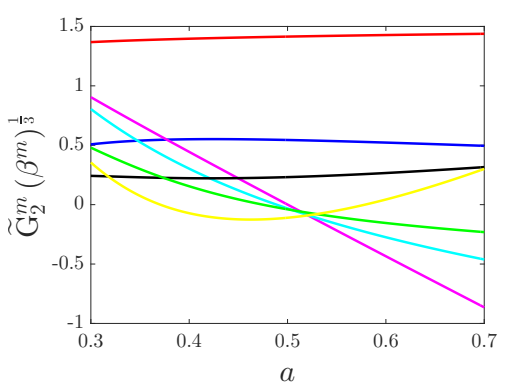

(b) Weighted $\widetilde{\mathrm{G}}_{2}^{m}$

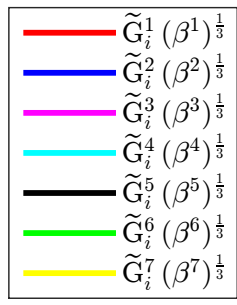

Fig. 6: Unit-cell: load case $X X$, normalized modal functions with PGD compression. 


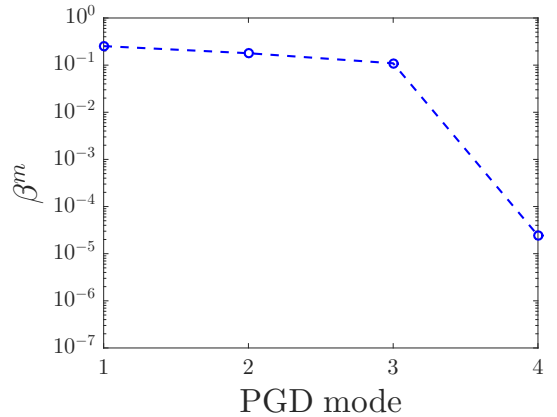

(a) No PGD compression

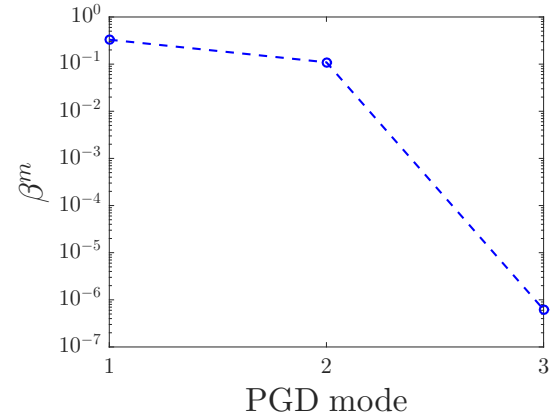

(b) With PGD compression

Fig. 7: Unit-cell: load case $Y Y$, evolution of modal amplitudes $\beta^{m}, m=$ $1,2, \ldots, n$.

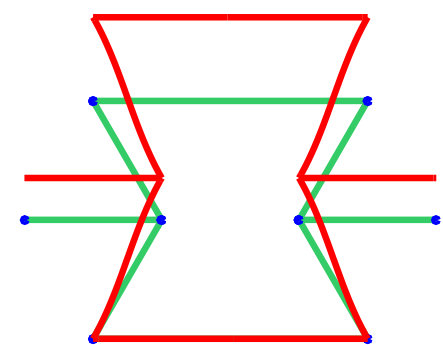

(a) Scaled $\widetilde{\mathbf{u}}^{1}$

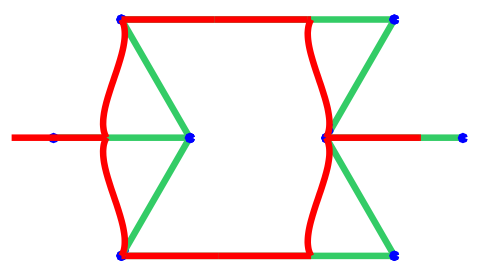

(b) Scaled $\widetilde{\mathbf{u}}^{2}$

Fig. 8: Unit-cell: load case $Y Y$, shape of the first modal vectors with PGD compression (in red). 


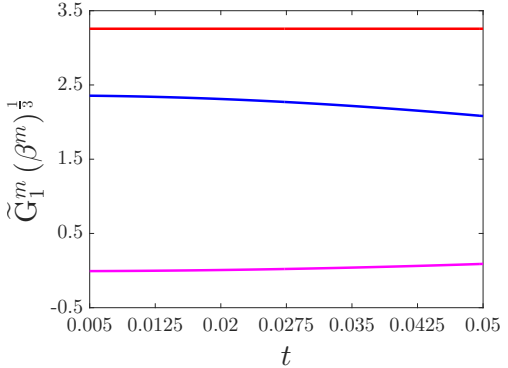

(a) Weighted $\widetilde{\mathrm{G}}_{1}^{m}$

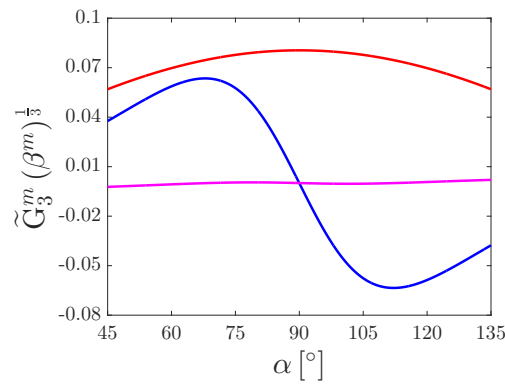

(c) Weighted $\widetilde{\mathrm{G}}_{3}^{m}$

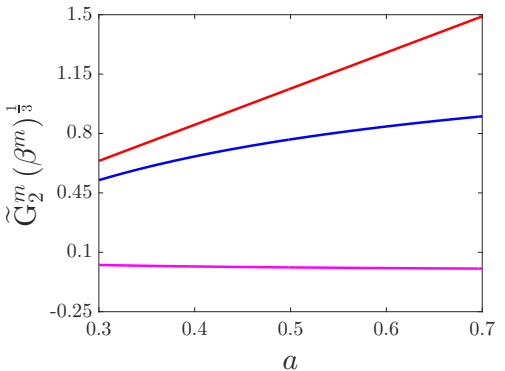

(b) Weighted $\widetilde{\mathrm{G}}_{2}^{m}$

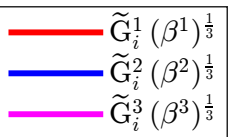

Fig. 9: Unit-cell: load case $Y Y$, normalized modal functions with PGD compression.

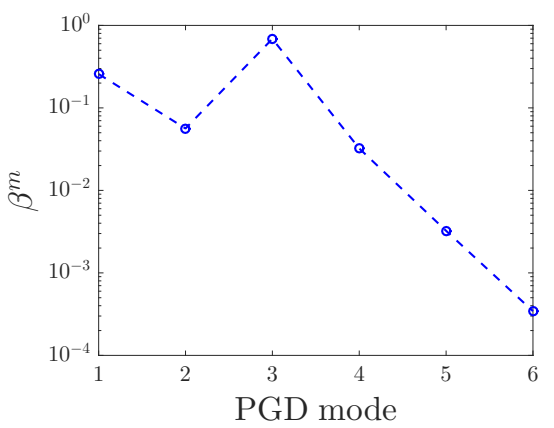

(a) No PGD compression

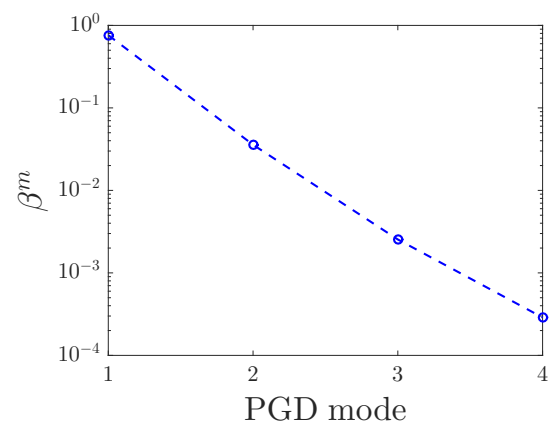

(b) With PGD compression

Fig. 10: Unit-cell: load case $X Y$, evolution of modal amplitudes $\beta^{m}, m=$ $1,2, \ldots, n$. 


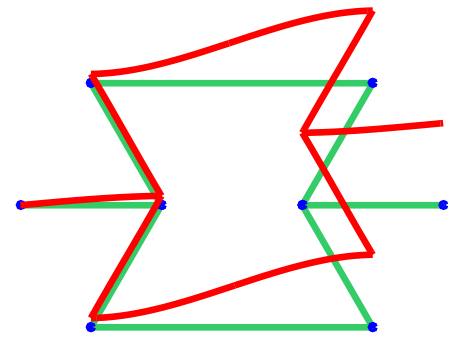

(a) Scaled $\widetilde{\mathbf{u}}^{1}$

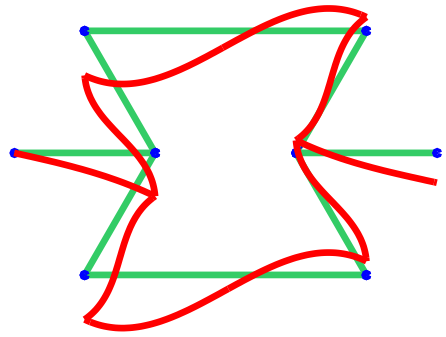

(b) Scaled $\widetilde{\mathbf{u}}^{3}$

Fig. 11: Unit-cell: load case $X Y$, shape of the first modal vectors with PGD compression (in red).

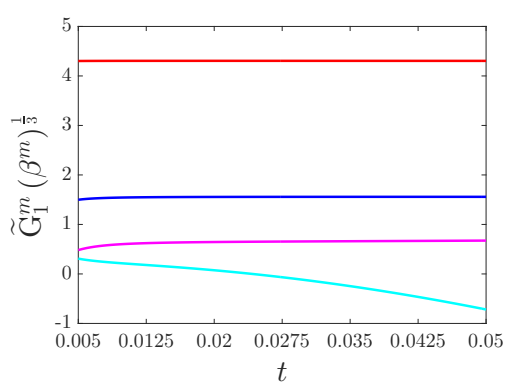

(a) Weighted $\widetilde{G}_{1}^{m}$

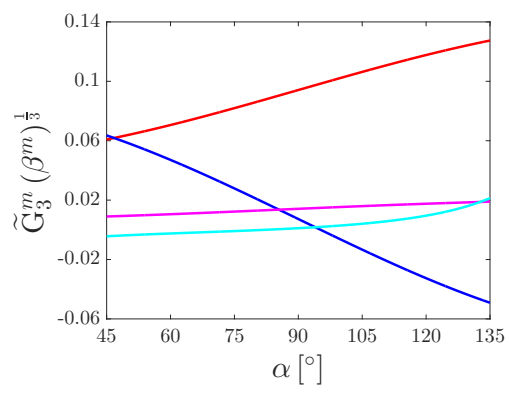

(c) Weighted $\widetilde{\mathrm{G}}_{3}^{m}$

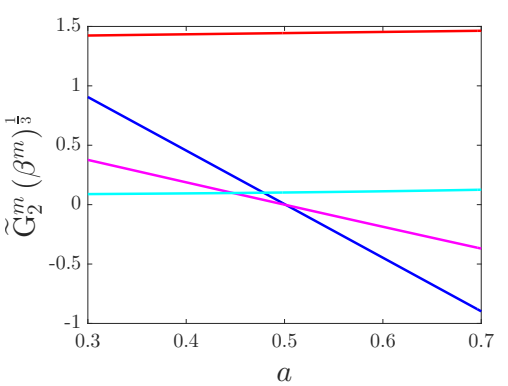

(b) Weighted $\widetilde{\mathrm{G}}_{2}^{m}$

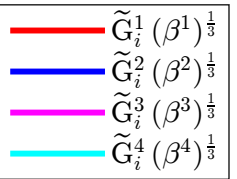

Fig. 12: Unit-cell: load case $X Y$, normalized modal functions with PGD compression. 
Figures 4, 5 and 6 refer to the load case $X X$. It is observed that in order to reach the desired accuracy, the original PGD solution requires 15 modes, which are compressed into 7, using the technique described in Section 3.5.

Figures 7, 8 and 9 refer to the load case $Y Y$, where the numbers of PGD terms is 4 without compression and 3 once compressed. In this case it is clearly seen that the second modal function in Figure 9c (blue color) governs the auxetic behaviour of the structured material for $\alpha<90^{\circ}$.

Figures 10, 11 and 12 illustrate the load case $X Y$, where the required number of modes is 6 and 4, for the raw PGD and the compressed one, respectively.

As shown in Appendix B, the analytical solution $\mathbf{U}(\boldsymbol{\mu})$ of the discrete system (1) in terms of the parameters $\mu_{i}$ is available. This is going to be used in the following to assess the convergence of $\mathbf{U}_{\mathrm{PGD}}^{n}(\boldsymbol{\mu})$, the proposed numerical solution using PGD with compression. In order to measure the error, the parametric solution is evaluated in the Cartesian domain $I_{1} \times I_{2} \times I_{3}$ using uniformly distributed points. For intervals $I_{1}$ and $I_{2}, 25$ points are used, while 125 points are used for $I_{3}$. Then, the relative error of generalized displacements is computed as:

$$
\epsilon_{\mathrm{r}}=\frac{\sum_{k=1}^{125} \sum_{j=1}^{25} \sum_{i=1}^{25}\left\|\mathbf{U}\left(\mu_{1}^{i}, \mu_{2}^{j}, \mu_{3}^{k}\right)-\mathbf{U}_{\mathrm{PGD}}^{n}\left(\mu_{1}^{i}, \mu_{2}^{j}, \mu_{3}^{k}\right)\right\|}{\sum_{k=1}^{125} \sum_{j=1}^{25} \sum_{i=1}^{25}\left\|\mathbf{U}\left(\mu_{1}^{i}, \mu_{2}^{j}, \mu_{3}^{k}\right)\right\|},
$$

where the superscripts $i, j$ and $k$ range the points in the domain where the multidimensional representation is sampled.

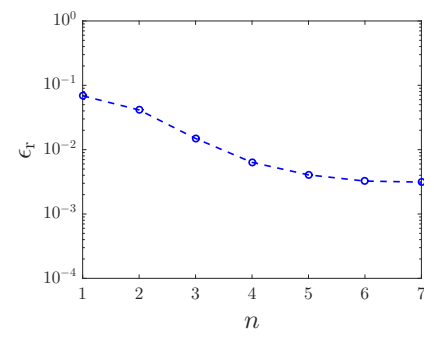

(a) Case $X X$

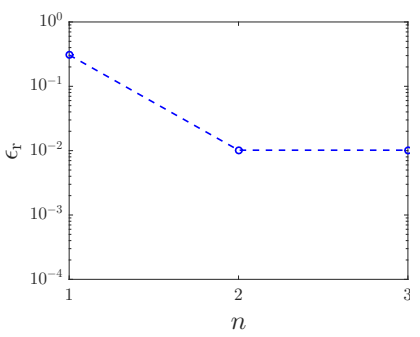

(b) Case $Y Y$

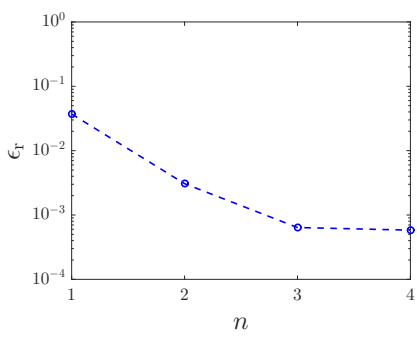

(c) Case $X Y$

Fig. 13: Relative error of $\mathbf{U}_{\mathrm{PGD}}^{n}(\boldsymbol{\mu})$ in the unit-cell depending on the $n$ number of PGD modes with compression.

The results of the convergence using equation (38) for the load cases $X X$, $Y Y$ and $X Y$ are shown in Figures 13a, 13b and 13c, respectively.

The components of the effective constitutive tensor can be constructed using the generalized displacement solutions of the unit-cell problem. For the 
present example, it can be shown that the effective mechanical properties result orthotropic, see Appendix B.

One of the most relevant mechanical properties in auxetic materials is the effective Poisson's ratio. Being the material sheet orthotropic, two different Poisson's ratios - $\nu_{12}^{\text {eff }}$ and $\nu_{21}^{\text {eff }}$ - are obtained using (36) and (37), as mentioned in [6], which assumes a plane stress state and reads:

$$
\begin{aligned}
& \nu_{12}^{\text {eff }}=\frac{\mathrm{C}_{12}^{\text {eff }}}{\mathrm{C}_{22}^{\text {eff }}} \text { and } \\
& \nu_{21}^{\text {eff }}=\frac{\mathrm{C}_{12}^{\text {eff }}}{\mathrm{C}_{11}^{\text {eff }}} .
\end{aligned}
$$

Therefore, in order to compute (39), the displacements solutions of the unit-cell problem for load cases $X X$ and $Y Y$ are needed.

In Figures 14a and 14b we can see the orthotropic Poisson's ratios for the parametric Cartesian domain given by $I_{2} \times I_{3}$, taking $t=\frac{1}{40}$. We fixed $t$ as a constant since $a$ and $\alpha$ parameters are highly more relevant for Poisson's ratio variations.

The represented response surfaces are computed using the PGD solutions for the unit-cell subjected to load cases $X X$ and $Y Y$. The approximations have 7 and 3 modes respectively, as shown when using PGD compression.

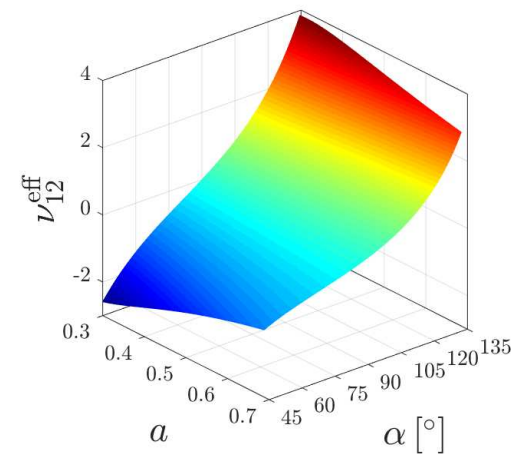

(a) $\nu_{12}^{\text {eff }}$

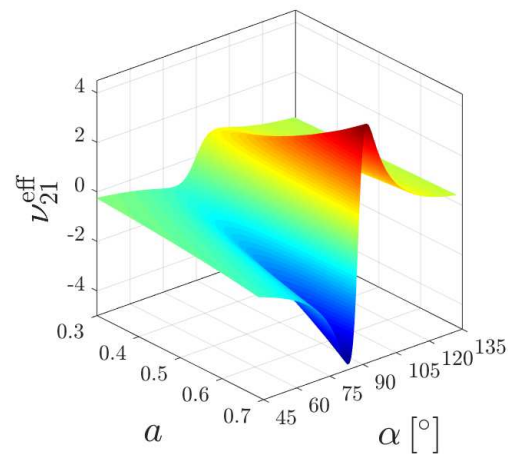

(b) $\nu_{21}^{\text {eff }}$

Fig. 14: Orthotropic effective Poisson's ratio using PGD.

Figure 14 shows very realistic and detailed response surfaces of the Poisson's ratio obtained by modifying parameters $a$ and $\alpha$, this latter being the most relevant parameter involved in auxetic behaviour. Furthermore, the normalized modal functions confirm such statement, including also the almost non-relevant dependence on thickness $t$. It is well-known that Poisson's ratio limits for isotropic materials are: $-1<\nu<0.5$. The values obtained for $\nu_{12}^{\text {eff }}$ 
and $\nu_{21}^{\text {eff }}$, as shown in Figure 14, do not completely verify this restriction. This is because, in the 2D-orthotropic setup, the thermodynamical consistency, see reference [19], is guaranteed by the conditions:

$$
\begin{aligned}
\nu_{12}^{\text {eff }} \nu_{21}^{\text {eff }} & >0 \text { and } \\
1-\nu_{12}^{\text {eff }} \nu_{21}^{\text {eff }} & >0,
\end{aligned}
$$

which are truly respected by the results displayed in Figure 14 .

In addition, in order to asses the error in the Poisson's ratios shown in Figure 14, an error $\varsigma_{\mathrm{r}}$ is computed in equation (40), in a similar fashion that in (38) but considering a Cartesian domain $I_{2} \times I_{3}$, with $\mu_{1}=\frac{1}{40}$. Therefore, the accuracy of the PGD orthotropic Poisson's ratio responses is compared against the analytical expressions obtained in Appendix B. It can be seen in Figure 15 a very good agreement in the orthotropic Poisson's ratios attained by PGD.

$$
\varsigma_{\mathrm{r}}=\frac{\sum_{j=1}^{125} \sum_{i=1}^{25}\left\|\nu_{\mathrm{PGD}}^{\text {eff }}\left(\mu_{1}=\frac{1}{40}, \mu_{2}^{i}, \mu_{3}^{j}\right)-\nu^{\text {eff }}\left(\mu_{1}=\frac{1}{40}, \mu_{2}^{i}, \mu_{3}^{j}\right)\right\|}{\sum_{j=1}^{125} \sum_{i=1}^{25}\left\|\nu^{\text {eff }}\left(\mu_{1}=\frac{1}{40}, \mu_{2}^{i}, \mu_{3}^{j}\right)\right\|},
$$

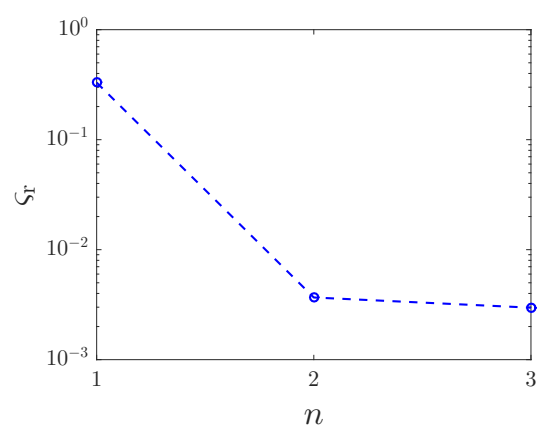

(a) Error $\varsigma_{r}$ for $\nu_{12 \mathrm{PGD}}^{\text {eff }}$

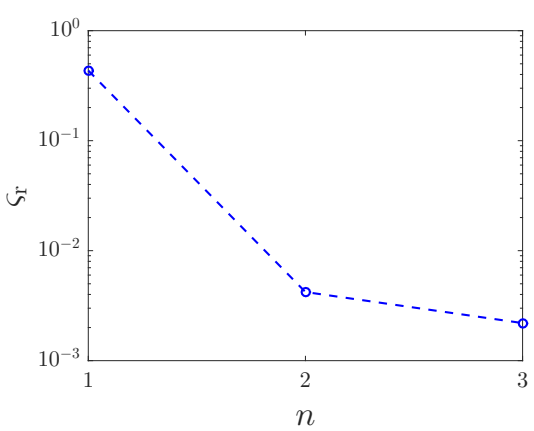

(b) Error $\varsigma_{\mathrm{r}}$ for $\nu_{21 \mathrm{PGD}}^{\text {eff }}$

Fig. 15: Unit-cell model relative error of Poisson's ratios between PGD vs analytical.

\subsection{Periodic pattern}

Here, the PGD approximation of the solution will be carried out in a periodic lattice material structure by taking into account the total number of degrees of freedom (non homogenized), instead of just a single cell. To do so, we use a periodic pattern made up from a total of $5 \times 5$ unit-cells. Two load cases are going to be considered: i) a uniaxial tensile test in $X$ direction (Figure 16) and ii) a unixial tensile in $Y$ direction (Figure 17). 


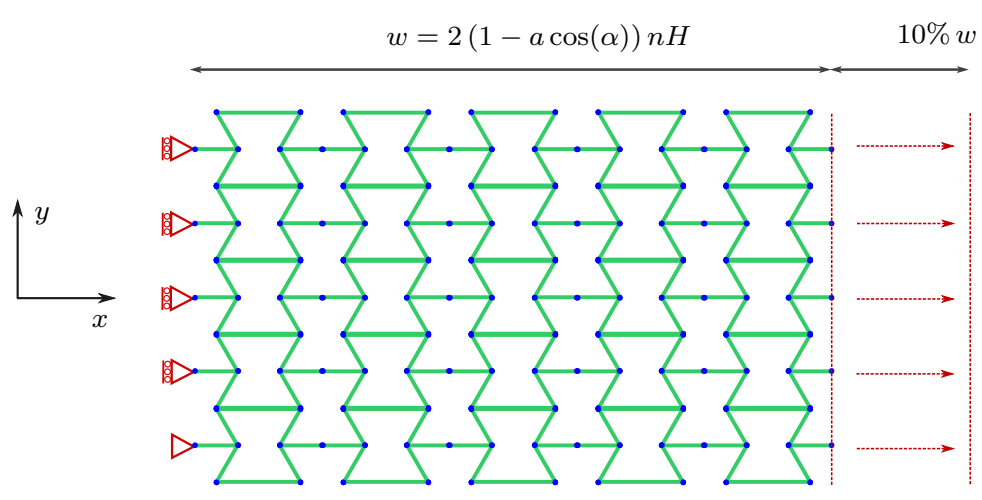

Fig. 16: Periodic array loaded in $X$ direction.

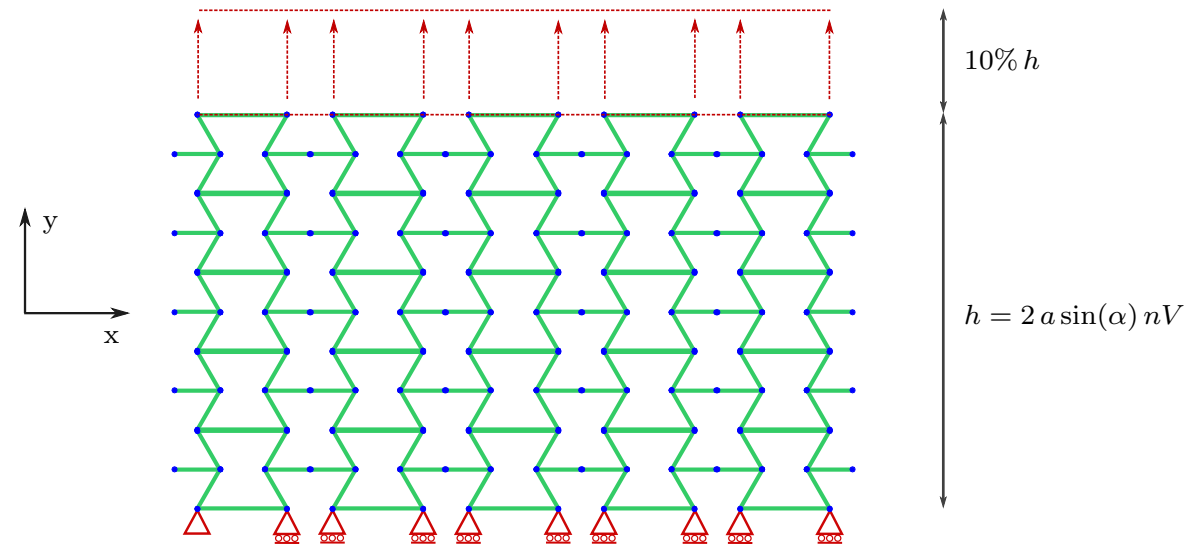

Fig. 17: Periodic array loaded in $Y$ direction.

The evolution of the modal amplitudes $\beta^{m}$ is plotted in Figures 18 and 19 for the load cases $X$ and $Y$ respectively. In addition, these figures compare how modal amplitudes evolve between the standard PGD greedy approach and the compressed PGD one. The latter scheme shows not only a significant reduction in modes but also an improved evolution in the decreasing tendency of the modal amplitudes.

Compared with the unit-cell model, the full pattern analysis gives the possibility of a better exploration of the parameters influence in a generic structured material. This is because the boundary effects are visible in this model, whereas in the homogenized one they are cancelled by the imposed periodic constraints. 


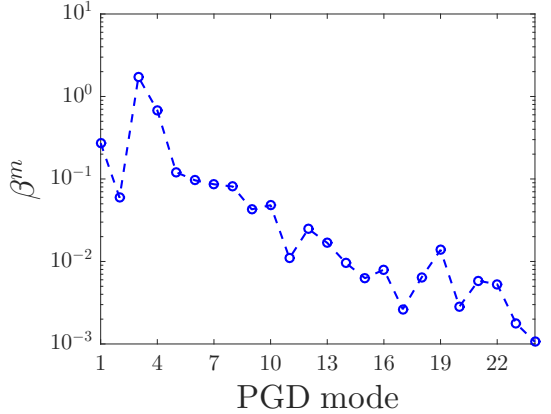

(a) No PGD compression

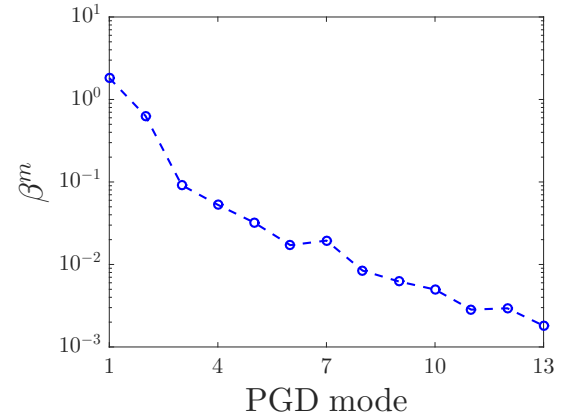

(b) With PGD compression

Fig. 18: Periodic pattern load case $X$, evolution of modal amplitudes.

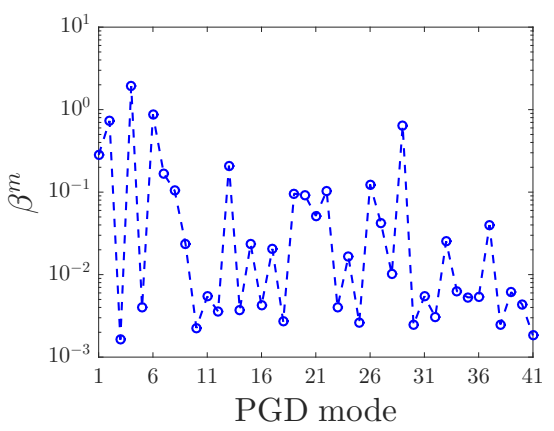

(a) No PGD compression

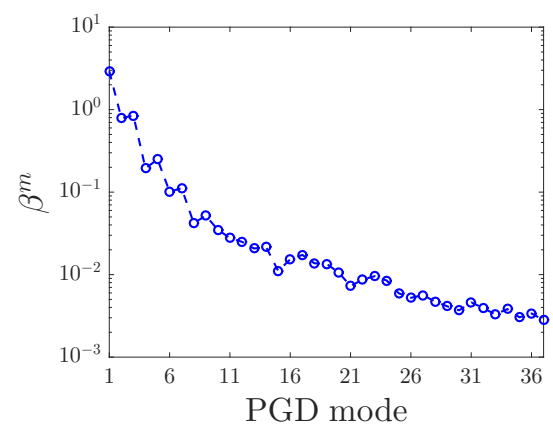

(b) With PGD compression

Fig. 19: Periodic pattern load case $Y$, evolution of modal amplitudes.

In the following, a measurement of the orthotropic Poisson's ratios in the full lattice material arrangement is proposed. Due to boundary effects, the Poisson's ratio is not uniform all along the sections of the structure. Therefore, it is obtained as an average of the transversal deformations in all the corresponding boundary nodes, see Figure 20 as an example for the load case $X$. The estimation of $\nu_{12 \mathrm{PGD}}^{\mathrm{pat}}$ is then computed as: 


$$
\begin{aligned}
\epsilon_{11} & =\frac{u(a, \alpha)}{w_{0}(a, \alpha)}=10 \%, \\
\epsilon_{22} & =\frac{h_{f}(t, a, \alpha)-h_{0}(a, \alpha)}{h_{0}(a, \alpha)}, \\
\nu_{12 \text { PGD }}^{\text {pat }} & =\frac{-\epsilon_{22}}{\epsilon_{11}}=-10 \epsilon_{22} .
\end{aligned}
$$

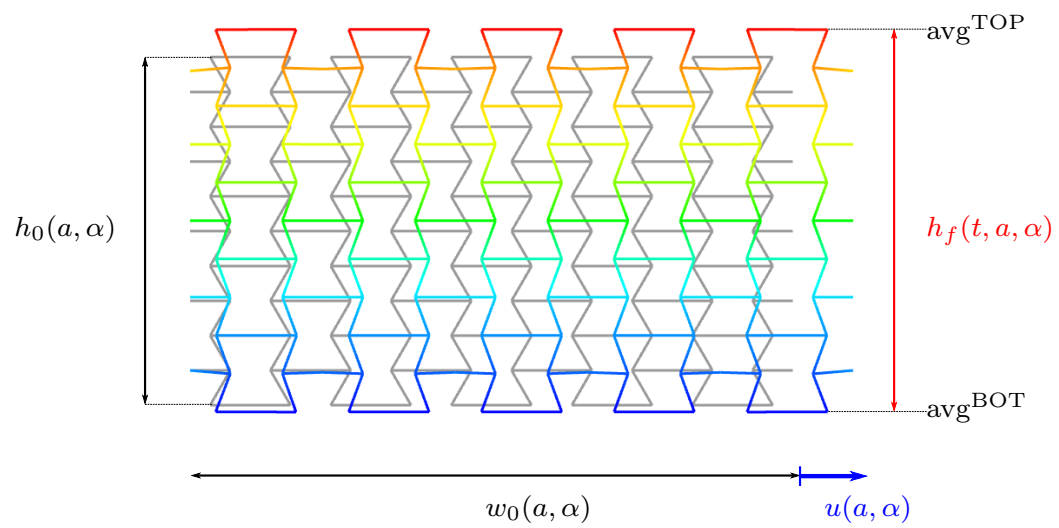

Fig. 20: Measurement of $\nu_{12 \text { PGD }}^{\text {pat }}$ in the periodic pattern subjected to load $X$.

The calculation of $\nu_{21 \mathrm{PGD}}^{\mathrm{pat}}$ is done analogously but using load case $Y$ instead. In Figure 21 it is shown the Poisson's ratios relative error $\varsigma_{\mathrm{r}}$, computed as reported in equation (40), but comparing here the full structure model against the homogenized one. Two different sizes have been considered for the full lattice material, one contains a total amount of $5 \times 5$ unit-cells whereas the other holds $10 \times 10$ unit-cells. 


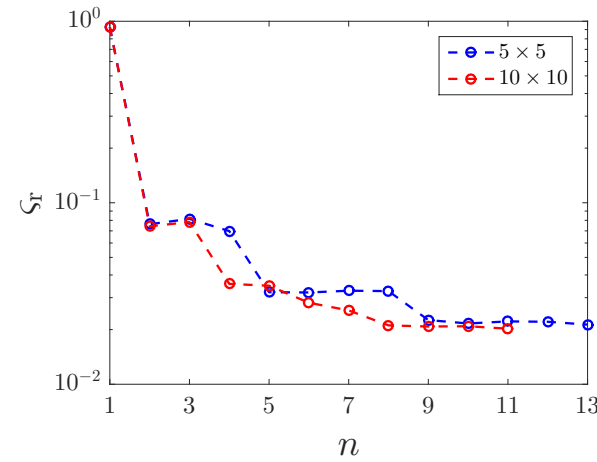

(a) Error $\varsigma_{\mathrm{r}}$ for $\nu_{12 \mathrm{PGD}}^{\mathrm{pat}}$

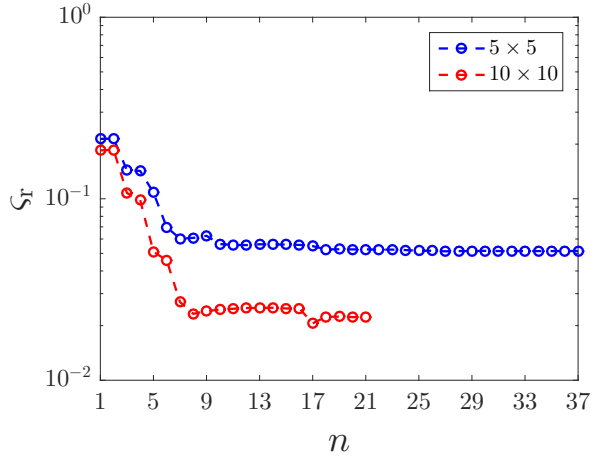

(b) Error $\varsigma_{\mathrm{r}}$ for $\nu_{21 \mathrm{PGD}}^{\mathrm{pat}}$

Fig. 21: Relative error of Poission's ratios computed in the full lattice material structure against the homogenized one.

As expected, Figure 21 suggests that the Poisson's ratios computed in the lattice material structure compare better to the homogenized ones as long as the number of unit-cells is increased in the full structure.

\subsection{Irregular pattern}

In this section, the same load cases as in Section 4.2 are shown, but in this example, the irregular pattern of Figure 22 will be used, for which its analytical solution is not available due to the high complexity of the parametric result.

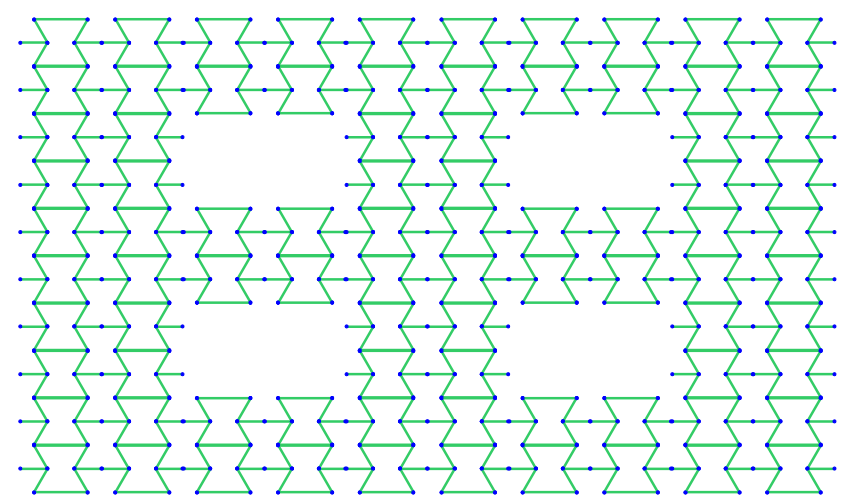

Fig. 22: 2D Architectured material with irregular pattern. 
The modal amplitudes using PGD compression can be seen in Figure 23. Results show that, as expected, the modal amplitudes decrease by increasing the number of PGD modes.

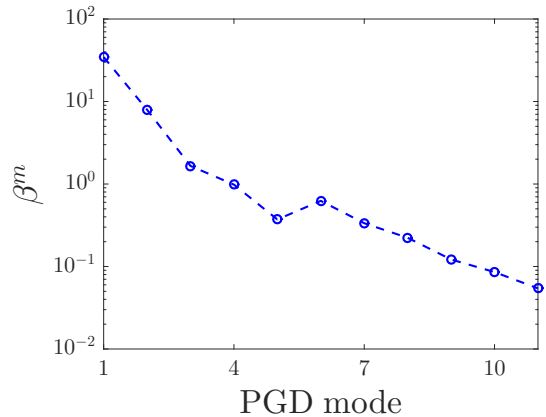

(a) Load case $X$

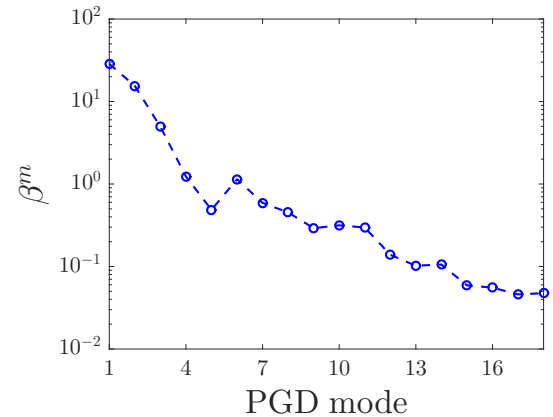

(b) Load case $Y$

Fig. 23: Modal amplitudes using PGD with compression for a non periodic structure pattern.

The present results show a similar global behaviour when compared to the periodic pattern, despite noticeable differences in the parametric modal functions. An example of this is depicted in Figure 24 for the normalized modal functions $\widetilde{\mathrm{G}}_{3}^{m}(\alpha)$. 


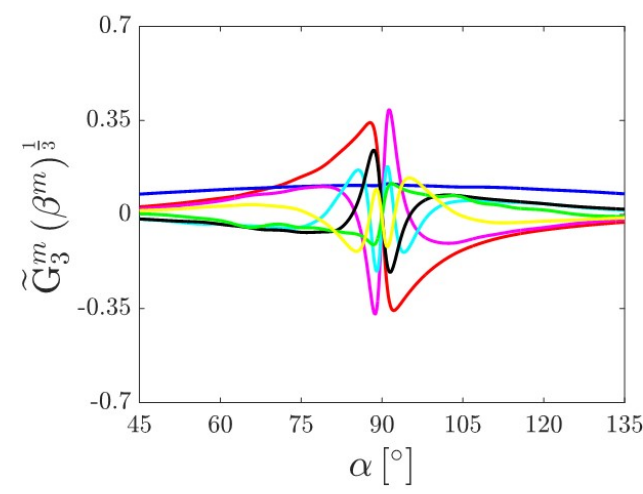

(a)

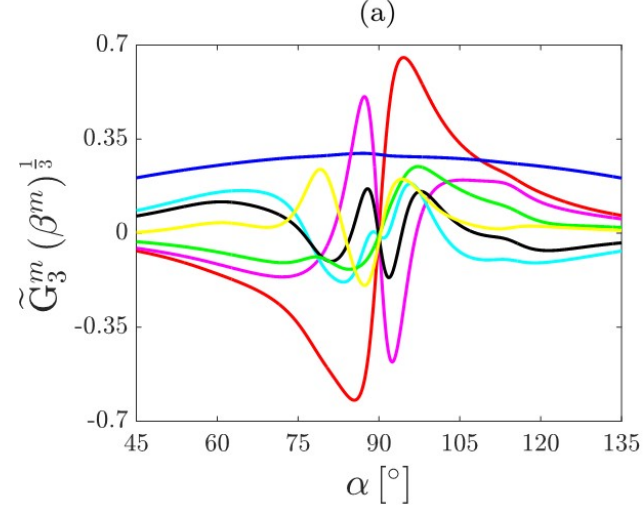

(b)

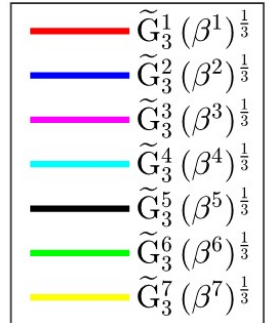

Fig. 24: Normalized modal functions $\widetilde{\mathrm{G}}_{3}^{m}(\alpha)$ obtained with PGD compression for load case $Y$ in the (a) periodic pattern and the (b) irregular pattern. A contrasting behaviour is observed in these oscillatory functions given the differences in the lattice material patterns.

\section{Concluding remarks}

The present paper presents in detail the generalization of PGD to parameterized structural problems. This tool is applied to model the macroscopic behavior of architectured materials, where the parameters describe the shape and structural properties of the microstructure. This allows explicitly reproducing the response surfaces for the quantities of interest (e.g., the different orthotropic Poisson's ratios if the goal is obtaining specific auxetic properties) in terms of the design parameters. In other words, the quantities of interest are explicitly represented by computational vademecums. Consequently, the inverse problem corresponding to optimal material design is solved as a trivial post-process. 
The 3D printing opens the possibility of manufacturing any of these materials for arbitrary values of the parameters and therefore this technique is currently extremely pertinent. Any optimal configuration proposed by the methodology can be easily brought into reality.

The possibility of explicitly representing the dependence of the macroscopic response as a function of the parameters describing the metamaterial cells is a promising tool. So far, the capabilities of this technology are demonstrated for $2 \mathrm{D}$ tessellations of seed cells with linear behavior. The extension to $3 \mathrm{D}$ complex structures is conceptually straightforward and opens the door to very interesting applications. Modeling the nonlinear regime is also important to properly describe the full range of applications. The nonlinear generalization is less obvious but deserves devoted research efforts.

Acknowledgements This work has been supported by Ministerio de Economía y Competitividad, grant numbers DPI2014-51844-C2-2-R and DPI2017-85139-C2-2-R, together with Generalitat de Catalunya, grant number 2014-SGR-1471 and European Education, Audiovisual and Culture Executive Agency (EACEA) under the Erasmus Mundus Joint Doctorate Simulation in Engineering and Entrepreneurship Development (SEED), FPA 2013-0043.

\section{References}

1. Almgren, R.F.: An isotropic three-dimensional structure with Poisson's ratio $=-1$. J. Elasticity 15(4), 427-430 (1985)

2. Amendola, A., Smith, C.J., Goodall, R., Auricchio, F., Feo, L., Benzoni, G., Fraternali, F.: Experimental response of additively manufactured metallic pentamode materials confined between stiffening plates. Compos. Struct. 142, 254-262 (2016)

3. Ammar, A., Chinesta, F., Díez, P., Huerta, A.: An error estimator for separated representations of highly multidimensional models. Computer Methods in Applied Mechanics and Engineering 199(25), 1872-1880 (2010)

4. Anthoine, A.: Derivation of the in-plane elastic characteristics of masonry through homogenization theory. Int. J. Solids Structures 32(2), 137-163 (1995)

5. Banerjee, D., Ji, C., Iizuka, H.: Invisibility cloak with image projection capability 6, 38,965 EP - (2016). URL http://dx.doi.org/10.1038/srep38965

6. Bauchau, O.A., Craig, J.I.: Structural analysis: with applications to aerospace structures, vol. 163. Springer Science \& Business Media (2009)

7. Bertoldi, K., Reis, P.M., Willshaw, S., Mullin, T.: Negative poisson's ratio behavior induced by an elastic instability. Advanced Materials 22(3), 361-366 (2010)

8. Cadman, J.E., Zhou, S., Chen, Y., Li, Q.: On design of multi-functional microstructural materials. Journal of Materials Science 48(1), 51-66 (2013)

9. Chinesta, F., Ammar, A., Cueto, E.: Recent advances and new challenges in the use of the proper generalized decomposition for solving multidimensional models. Archives of Computational methods in Engineering 17(4), 327-350 (2010)

10. Christensen, J., Kadic, M., Kraft, O., Wegner, M.: Vibrant times for mechanical metamaterials. MRS Communications 5, 453-462 (2015)

11. Cook, R.D., Malkus, D.S., Plesha, M.E., Witt, R.J.: Concepts and Applications of Finite Element Analysis, fourth edn. John Wiley \& Sons, Inc., USA (2002)

12. Elipe, J.C.Á., Lantada, A.D.: Comparative study of auxetic geometries by means of computer-aided design and engineering. Smart Materials and Structures 21(10), 105,004 (2012)

13. Ergin, T., Stenger, N., Brenner, P., Pendry, J.B., Wegener, M.: Three-Dimensional Invisibility Cloack at Optical Wavelengths. Science 328, 337-339 (2010)

14. Greaves, G.N., Greer, A.L., Lakes, R.S., Rouxel, T.: Poisson's ratio and modern materials. Nat. Mater. 10(11), 823837 (2011) 
15. Hopkins, J.B., Lange, K.J., Spadaccini, C.M.: Designing Microstructural Architectures With Thermally Actuated Properties Using Freedom, Actuation and Constraint Topologies. Journal of Mechanical Design. ASME 135(6), 061,004 (2013)

16. Kadic, M., Bückmann, T., Schittny, R., Wegener, M.: Metamaterials beyond electromagnetism. Reports on Progress in Physics 76(12), 126,501 (2013)

17. Kadic, M., Bückmann, T., Stenger, N., Thiel, M., Wegener, M.: On the practicability of pentamode mechanical metamaterials. Appl. Phys. Lett. 100(19), 191,901 (2012)

18. Lantada, A.D., Muslija, A., García-Ruíz, J.P.: Auxetic tissue engineering scaffolds with nanometric features and resonances in the megahertz range. Smart Materials and Structures 24(5), 055,013 (2015)

19. Lempriere, B.: Poissons ratio in orthotropic materials. AIAA J 6(11), 2226-2227 (1968)

20. Michel, J.C., Moulinec, H., Suquet, P.: Effective properties of composite materials with periodic microstructure: a computational approach. Comput. Methods Appl. Mech. Engrg. 172, 109-143 (1999)

21. Milton, G.W., Cherkaev, A.: Which Elasticity Tensors are Realizable? J. Eng. Mater-T. ASME 117, 483-493 (1995)

22. Modesto, D., Zlotnik, S., Huerta, A.: Proper Generalized Decomposition for parameterized helmholtz problems in heterogeneous and unbounded domains: application to harbor agitation. Comput. Methods Appl. Mech. Eng. 295, 127-149 (2015). DOI 10.1016/j.cma.2015.03.026

23. Rafsanjani, A., Akbarzadeh, A., Pasini, D.: Snapping Mechanical Metamaterials under Tension. Adv. Mater. 27(39), 5931-5935 (2015)

24. Rafsanjani, A., Pasini, D.: Bistable auxetic mechanical metamaterials inspired by ancient geometric motifs. Extreme Mechanics Letters 9, 291-296 (2016)

25. Reis, P.M.: A Perspective on the Revival of Structural (In) Stability With Novel Opportunities for Function: From Buckliphobia to Buckliphilia. J. Appl. Mech-T. ASME 82, 111,001 (2015)

26. Reis, P.M., Jaeger, H.M., van Hecke, M.: Designer Matter: A perspective. Extreme Mechanics Letters 5, 25-29 (2015)

27. Sigmund, O.: Design of material structures using topology optimization. Ph.D. thesis, Technical University of Denmark (1994)

28. Sigmund, O.: Materials with prescribed constitutive parameters: An inverse homogenization problem. I. J. Solids Struct. 31(17), 2313-2329 (1994)

29. Sigmund, O.: Tailoring materials with prescribed elastic properties. Mech. Mater. 20, 351-368 (1995)

30. Sigmund, O., Torquato, S.: Composites with extremal thermal expansion coefficients. Appl. Phys. Lett. 69(21), 3203-3205 (1996)

31. Zlotnik, S., Díez, P., González, D., Cueto, E., Huerta, A.: Effect of the separated approximation of input data in the accuracy of the resulting PGD solution. Adv. Model. Simul. Eng. Sci. 2(28) (2015). DOI 10.1016/j.cma.2015.03.026

32. Zlotnik, S., Díez, P., Modesto, D., Huerta, A.: Proper generalized decomposition of a geometrically parametrized heat problem with geophysical applications. International Journal for Numerical Methods in Engineering 103(10), 737-758 (2015) 


\section{A . Proper Generalized Decomposition Algorithms}

Data: $\mathbf{K}^{k}, B_{i}^{k}, \mathbf{f}^{\ell}, S_{i}^{\ell}$ and $\mathbf{U}_{\mathrm{PGD}}^{n-1}(\boldsymbol{\mu}): \mathbf{u}^{m}, G_{i}^{m}$, for $m=1,2, \ldots, n-1$, $k=1,2, \ldots, \mathrm{n}_{\mathrm{k}}, \ell=1,2, \ldots, \mathrm{n}_{\mathrm{f}}$

Result: $\mathbf{u}$ and $G_{i}$, for $i=1,2, \ldots, \mathrm{n}_{\mathrm{p}}$

Initialize: assign values to $\mathbf{u}$ and $G_{i}$; select a tolerance $\eta_{\text {tol }}$

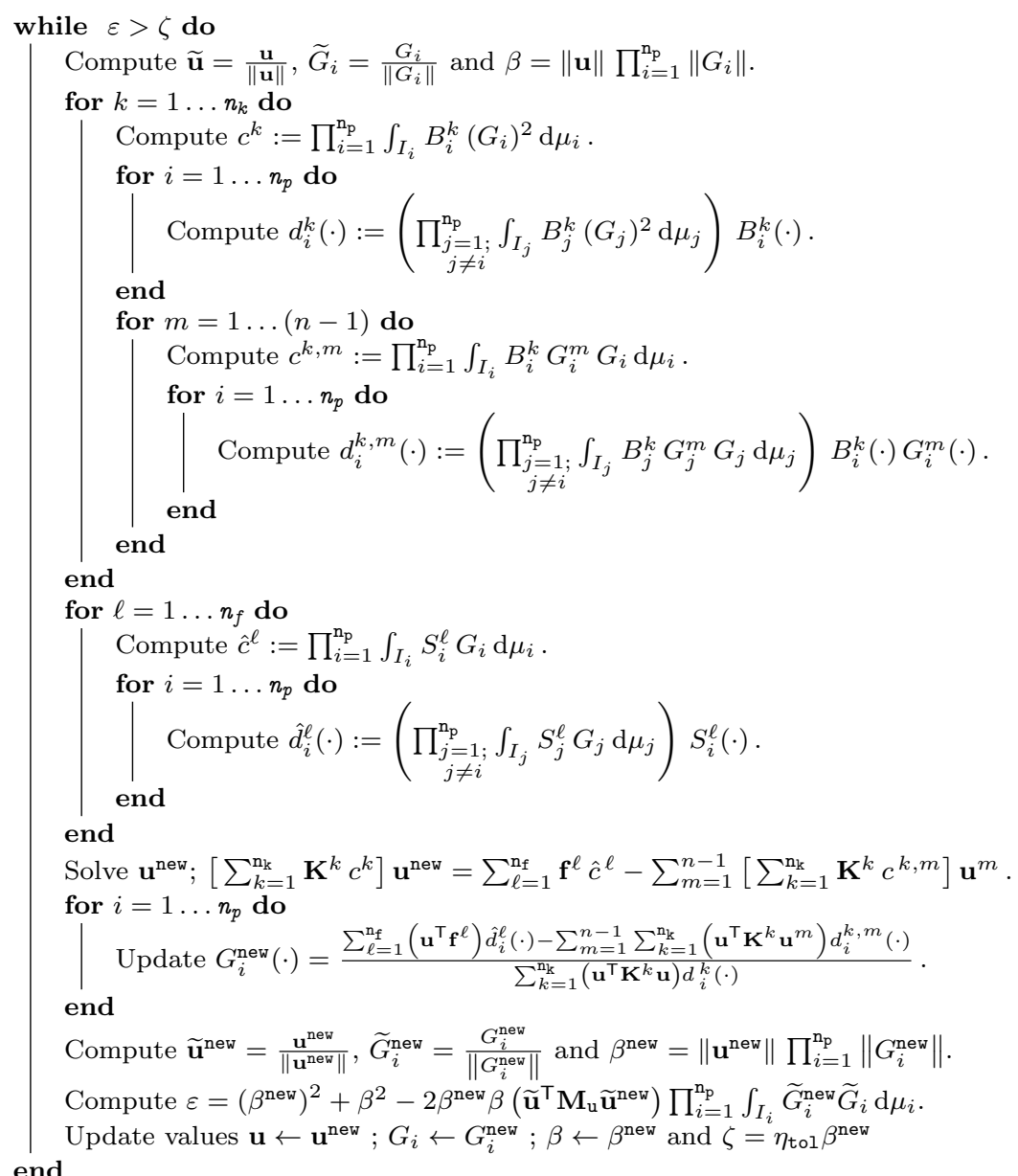

Algorithm 1: PGD alternated directions nonlinear solver 
Data: $\mathbf{U}_{\mathrm{PGD}}^{n}(\boldsymbol{\mu}): \mathbf{u}^{m}, G_{i}^{m}$, for $m=1,2, \ldots, n$ $\mathbf{U}_{\text {com }}^{\widehat{n}-1}: \widehat{\mathbf{u}}^{\widehat{m}}, \widehat{G}_{i}^{\hat{m}}$, for $\hat{m}=1,2, \ldots,(\widehat{n}-1)$

Result: $\widehat{\mathbf{u}} \widehat{G}_{i}$, for $i=1,2, \ldots, \mathrm{n}_{\mathrm{p}}$

Initialize: assign values to $\widehat{\mathbf{u}}$ and $\widehat{G}_{i}$; select a tolerance $\eta_{\mathrm{tol}}$

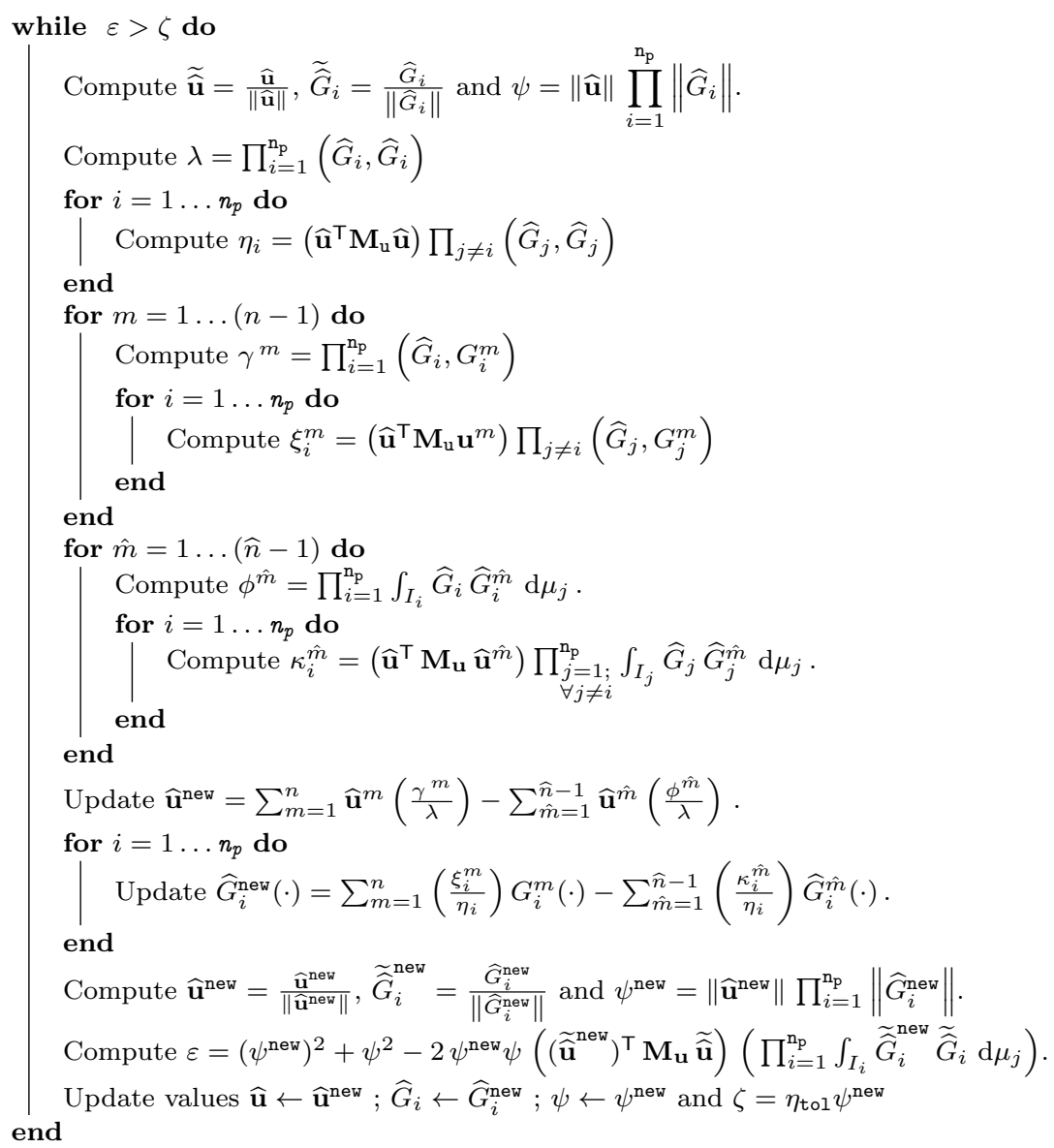

Algorithm 2: Least-Squares PGD alternated directions nonlinear solver

\section{B . Analytical solutions}

Given the unit-cell global stiffness matrix built by Bernoulli beam elements, $\mathbf{K}(\boldsymbol{\mu})$, the equations

$$
\begin{aligned}
\mathbf{K}(\boldsymbol{\mu}) \mathbf{U}(\boldsymbol{\mu}) & =\mathbf{0}, \\
\mathbf{C} \mathbf{U}(\boldsymbol{\mu}) & =\mathbf{Q}(\boldsymbol{\mu}),
\end{aligned}
$$

contain equilibrium and periodicity conditions, given a specific load case in the unit-cell. If the constraints are applied using a direct method [11], then the system unknowns can be split into released and constrained such as 


$$
\left[\begin{array}{ll}
\mathbf{C}_{\mathrm{R}} & \mathbf{C}_{\mathrm{C}}
\end{array}\right]\left\{\begin{array}{l}
\mathbf{U}_{\mathrm{R}}(\boldsymbol{\mu}) \\
\mathbf{U}_{\mathrm{C}}(\boldsymbol{\mu})
\end{array}\right\}-\{\mathbf{Q}(\boldsymbol{\mu})\}=\{\mathbf{0}\}
$$

in a way that the constrained unknowns can be solved in terms of the released

$$
\left\{\begin{array}{c}
\mathbf{U}_{\mathrm{R}}(\boldsymbol{\mu}) \\
\mathbf{U}_{\mathrm{C}}(\boldsymbol{\mu})
\end{array}\right\}=\underbrace{\left[\begin{array}{c}
\mathbf{I} \\
-\mathbf{C}_{\mathrm{C}}^{-1} \mathbf{C}_{\mathrm{R}}
\end{array}\right]}_{\boldsymbol{\varphi}}\left\{\mathbf{U}_{\mathrm{R}}(\boldsymbol{\mu})\right\}+\underbrace{\left\{\begin{array}{c}
\mathbf{0} \\
\mathbf{C}_{\mathrm{C}}^{-1} \mathbf{Q}(\boldsymbol{\mu})
\end{array}\right\}}_{\mathbf{Q}_{0}(\boldsymbol{\mu})} .
$$

The system can be solved for the released unknowns only:

$$
\mathbf{K}_{\mathrm{R}}(\boldsymbol{\mu}) \mathbf{U}_{\mathrm{R}}(\boldsymbol{\mu})=\mathbf{F}_{\mathrm{R}}((\mu)),
$$

where

$$
\begin{aligned}
\mathbf{K}_{\mathrm{R}}(\boldsymbol{\mu}) & =\boldsymbol{\varphi}^{\mathrm{T}} \mathbf{K}(\boldsymbol{\mu}) \boldsymbol{\varphi}, \\
\mathbf{F}(\boldsymbol{\mu})_{\mathrm{R}} & =-\boldsymbol{\varphi}^{\mathrm{T}} \mathbf{K}(\boldsymbol{\mu}) \mathbf{Q}_{0}(\boldsymbol{\mu}) .
\end{aligned}
$$

While $\forall \boldsymbol{\mu} \in \mathcal{D}=I_{1} \times I_{2} \times \ldots \times I_{\mathrm{n}_{\mathrm{p}}}, \mathbf{K}(\boldsymbol{\mu}) \in \mathbb{R}^{\left(\mathrm{n}_{\mathrm{dof}} \times \mathrm{n}_{\mathrm{dof}}\right)}, \mathbf{K}_{\mathrm{R}}(\boldsymbol{\mu}) \in \mathbb{R}^{\left(n_{\mathrm{R}} \times n_{\mathrm{R}}\right)}$. In the unitcell problem, $\mathrm{n}_{\mathrm{dof}}=24$ is reduced to $n_{\mathrm{R}}=13$. Now the system can be solved symbolically with the parameters $\boldsymbol{\mu}=\left[\begin{array}{llll}t & a & b & \alpha\end{array}\right]^{\top}$.

With this, the parametric solution for case $X X$ results:

$$
\mathbf{U}_{\mathrm{R}}(\boldsymbol{\mu})=\left\{\begin{array}{c}
u_{2} \\
v_{2} \\
\theta_{2} \\
u_{3} \\
v_{3} \\
\theta_{3} \\
u_{4} \\
v_{4} \\
\theta_{4} \\
u_{5} \\
v_{5} \\
\theta_{5} \\
\theta_{6}
\end{array}\right\}=\left\{\begin{array}{c}
\frac{b(a \cos (\alpha)-b)\left(\left(a^{2}-4 t^{2}\right) \sin ^{2}(\alpha)-a^{2}\right)}{a^{2}(a+2 b)-2 b\left(a^{2}-4 t^{2}\right) \sin ^{2}(\alpha)} \\
0 \\
0 \\
-\frac{(a \cos (\alpha)-b)\left(a^{2}(a+b)-b\left(a^{2}-4 t^{2}\right) \sin ^{2}(\alpha)\right)}{a^{2}(a+2 b)-2 b\left(a^{2}-4 t^{2}\right) \sin ^{2}(\alpha)} \\
0 \\
0 \\
-\frac{(a \cos (\alpha)-b)\left(a^{2}(a+3 b)-3 b\left(a^{2}-4 t^{2}\right) \sin ^{2}(\alpha)\right)}{a^{2}(a+2 b)-2 b\left(a^{2}-4 t^{2}\right) \sin ^{2}(\alpha)} \\
0 \\
0 \\
-\frac{(a \cos (\alpha)-b)\left(a^{2}(2 a+3 b)-3 b\left(a^{2}-4 t^{2}\right) \sin ^{2}(\alpha)\right)}{a^{2}(a+2 b)-2 b\left(a^{2}-4 t^{2}\right) \sin ^{2}(\alpha)} \\
0 \\
0 \\
0
\end{array}\right\} .
$$

Figure 25 shows a scaled analytical solution for case $X X$, evaluated at parameters $b=1$, $t=\frac{1}{40}, a=0.5, \alpha=60^{\circ}$ as an example.

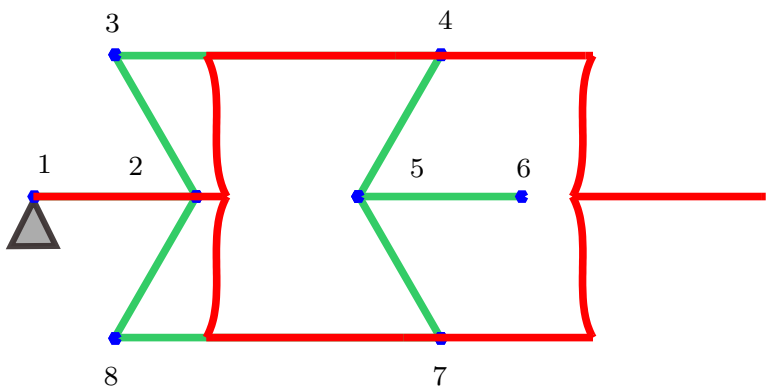

Fig. 25: Scaled generalized displacement solution case $X X$. 
The solution for load case $Y Y$ becomes:

$$
\mathbf{U}_{\mathrm{R}}(\boldsymbol{\mu})=\left\{\begin{array}{c}
u_{2} \\
v_{2} \\
\theta_{2} \\
\theta_{3} \\
u_{4} \\
v_{4} \\
\theta_{4} \\
u_{5} \\
v_{5} \\
\theta_{5} \\
u_{6} \\
v_{6} \\
\theta_{6}
\end{array}\right\}=\left\{\begin{array}{c}
-\frac{2 a b\left(a^{2}-4 t^{2}\right) \cos (\alpha) \sin ^{2}(\alpha)}{a^{3}+a^{2} b+4 b t^{2}+b\left(a^{2}-4 t^{2}\right) \cos (2 \alpha)} \\
a \sin (\alpha) \\
0 \\
0 \\
-\frac{2 a b\left(a^{2}-4 t^{2}\right) \cos (\alpha) \sin ^{2}(\alpha)}{a^{3}+a^{2} b+4 b t^{2}+b\left(a^{2}-4 t^{2}\right) \cos (2 \alpha)} \\
2 a \sin (\alpha) \\
0 \\
0 \\
a \sin (\alpha) \\
0 \\
-\frac{a b\left(a^{2}-4 t^{2}\right) \cos (\alpha) \sin ^{2}(\alpha)}{a^{3}+a^{2} b+4 b t^{2}+b\left(a^{2}-4 t^{2}\right) \cos (2 \alpha)} \\
a \sin (\alpha) \\
0
\end{array}\right\} .
$$

Figure 26 shows a scaled analytical solution for case $Y Y$, evaluated at parameters $b=1$, $t=\frac{1}{40}, a=0.5, \alpha=60^{\circ}$.

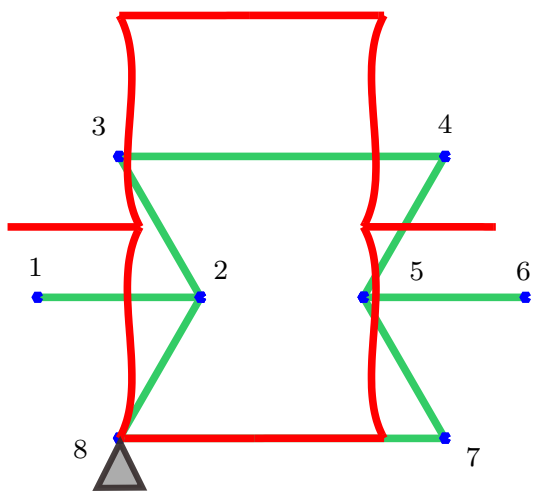

Fig. 26: Scaled generalized displacement solution case $Y Y$. 
Finally, for case $X Y$ :

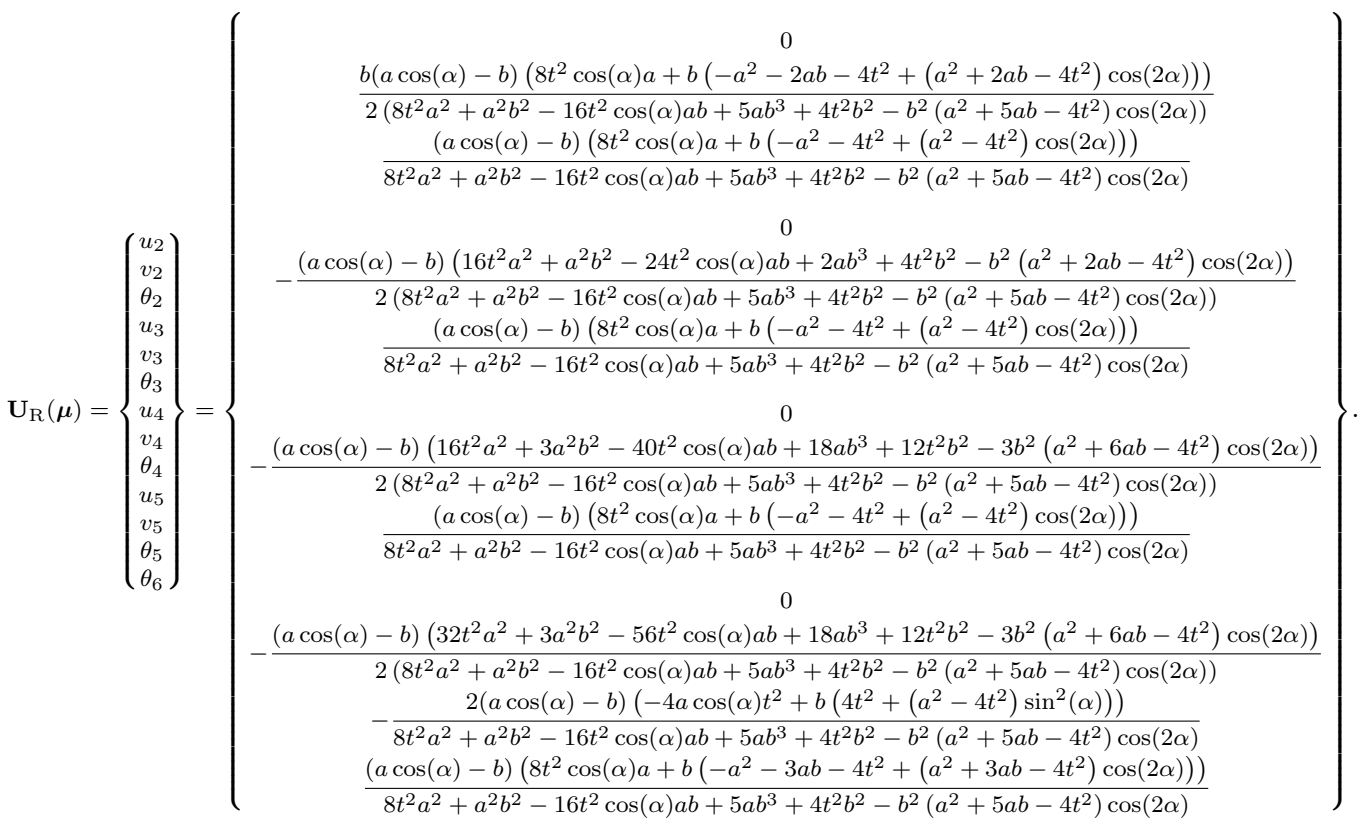

Figure 27 shows a scaled analytical solution for case $X Y$, evaluated at parameters $b=1$, $t=\frac{1}{40}, a=0.5, \alpha=60^{\circ}$.

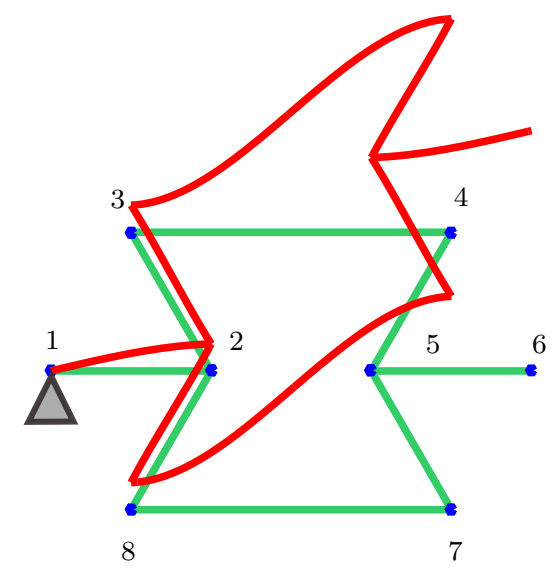

Fig. 27: Scaled generalized displacement solution case $X Y$.

At this point, it seems worth mentioning the parametric analytical solutions have the size of a unit-cell reduced by its periodic constraints. For a full structure done by repetitions of unit-cells, or a pattern with irregularities, the cost of obtaining such expressions becomes computationally unfeasible. 
Finally, the components of the effective elasticity matrix are calculated from (37) and are shown in equations (43) to (48). It is worth mentioning that these results are affected by a factor of $E$, the Young's modulus of the constituent material, which is here omitted for the sake of clarity:

$$
\begin{aligned}
& \mathrm{C}_{11}^{\text {eff }}(\boldsymbol{\mu})=-\frac{t \csc (\alpha)(a \cos (\alpha)-b)\left(\left(a^{2}-4 t^{2}\right) \cos (2 \alpha)+a^{2}+4 t^{2}\right)}{a\left(a^{3}+b\left(a^{2}-4 t^{2}\right) \cos (2 \alpha)+a^{2} b+4 t^{2} b\right)} \\
& \mathrm{C}_{22}^{\text {eff }}(\boldsymbol{\mu})=-\frac{t \sin (\alpha)\left(a^{3}-a\left(a^{2}-4 t^{2}\right) \cos (2 \alpha)+4 a t^{2}+16 t^{2} b\right)}{(a \cos (\alpha)-b)\left(a^{3}+b\left(a^{2}-4 t^{2}\right) \cos (2 \alpha)+a^{2} b+4 t^{2} b\right)} \\
& \mathrm{C}_{33}^{\text {eff }}(\boldsymbol{\mu})=-\frac{16 t^{3} \sin (\alpha)(a \cos (\alpha)-b)}{-b^{2}\left(a^{2}+5 a b-4 t^{2}\right) \cos (2 \alpha)+8 a^{2} t^{2}+a^{2} b^{2}-16 a b t^{2} \cos (\alpha)+5 a b^{3}+4 t^{2} b^{2}} \\
& \mathrm{C}_{12}^{\text {eff }}(\boldsymbol{\mu})=\frac{t\left(4 t^{2}-a^{2}\right) \sin (2 \alpha)}{a^{3}+b\left(a^{2}-4 t^{2}\right) \cos (2 \alpha)+a^{2} b+4 t^{2} b} \\
& \mathrm{C}_{13}^{\text {eff }}(\boldsymbol{\mu})=0 \\
& \mathrm{C}_{23}^{\text {eff }}(\boldsymbol{\mu})=0
\end{aligned}
$$

Using (43), (44) and (46) the orthotropic Poisson's ratios can be calculated as follows, which assumes a state of plane stress ([6]):

$$
\begin{aligned}
\nu_{12}^{\text {eff }}(\boldsymbol{\mu}) & =\frac{\mathrm{C}_{12}^{\text {eff }}(\boldsymbol{\mu})}{\mathrm{C}_{22}^{\text {eff }}(\boldsymbol{\mu})}=\frac{2\left(a^{2}-4 t^{2}\right) \cos (\alpha)(a \cos (\alpha)-b)}{a^{3}-a\left(a^{2}-4 t^{2}\right) \cos (2 \alpha)+4 a t^{2}+16 t^{2} b} \\
\nu_{21}^{\text {eff }}(\boldsymbol{\mu}) & =\frac{\mathrm{C}_{12}^{\text {eff }}(\boldsymbol{\mu})}{\mathrm{C}_{11}^{\text {eff }}(\boldsymbol{\mu})}=\frac{a\left(a^{2}-4 t^{2}\right) \sin (\alpha) \sin (2 \alpha)}{(a \cos (\alpha)-b)\left(\left(a^{2}-4 t^{2}\right) \cos (2 \alpha)+a^{2}+4 t^{2}\right)}
\end{aligned}
$$

